\title{
DISCRETIZATION METHODS WITH ANALYTICAL CHARACTERISTIC METHODS FOR ADVECTION-DIFFUSION-REACTION EQUATIONS AND 2D APPLICATIONS
}

\author{
Jürgen GeIser ${ }^{1}$
}

\begin{abstract}
Our studies are motivated by a desire to model long-time simulations of possible scenarios for a waste disposal. Numerical methods are developed for solving the arising systems of convectiondiffusion-dispersion-reaction equations, and the received results of several discretization methods are presented. We concentrate on linear reaction systems, which can be solved analytically. In the numerical methods, we use large time-steps to achieve long simulation times of about 10000 years. We propose higher-order discretization methods, which allow us to use large time-steps without losing accuracy. By decoupling of a multi-physical and multi-dimensional equation, simpler physical and one-dimensional equations are obtained and can be discretized with higher-order methods. The results can then be coupled with an operator-splitting method. We discuss benchmark problems given in the literature and real-life applications. We simulate a radioactive waste disposals with underlying flowing groundwater. The transport and reaction simulations for the decay chains are presented in $2 \mathrm{~d}$ realistic domains, and we discuss the received results. Finally, we present our conclusions and ideas for further works.
\end{abstract}

Mathematics Subject Classification. 35K15, 35K57, 47F05, 65M60, 65N30.

Received November 2nd, 2007. Revised October 25, 2008.

Published online August 1st, 2009.

\section{INTRODUCTION}

Our studies are motivated by a desire to model the transport of radioactive and chemical contaminants through an overlying rock. We are interested, in particular, in large-scale computations for long-term contamination in underlying porous media, see [14]. The mathematical model provides us with a coupled system of convection-diffusion-dispersion-reaction equations. To discretize such conservation law systems, finite volume methods are a class of discretization schemes that have been proven to be highly successful in approximating the solution (see [8-10]). Taking into account the different scales of such equations, we deal with adapted discretization and solver methods, see [25]. A particular idea is to split the full equations and solve each simpler equation in the adapted timescale. Here a time-splitting error has to be considered for such methods, Lie-Trotter and Strang splitting methods, see [29]. To overcome this problem, we have to apply higher-order

\footnotetext{
Keywords and phrases. Advection-diffusion-reaction equation, embedded analytical solutions, operator-splitting methods, characteristic methods, finite-volume methods, multi-physics, simulation of radioactive waste disposals

${ }^{1}$ Humboldt-Universität zu Berlin, Unter den Linden 6, 10099 Berlin, Germany. geiser@mathematik.hu-berlin.de
} 
splitting methods and solve equations partly analytically. Our contribution is to solve the convection and reaction equation together and embed an analytical one-dimensional test-function for the finite volume method. We apply and develop improved discretization and solver algorithms to achieve our target of higher efficiency and greater accuracy. The reaction equations as well as the convection equations belong to a small timescale and we use explicit temporal discretization. The discretization methods are derived for the convection-reaction equation with respect to finite volume and characteristic methods, see [1,6]. A modified discretization method is presented by embedding analytical one-dimensional solutions in multi-dimensional finite volume methods. The idea is based on Godunov's methods, see [20]. By decoupling the multi-dimensional problem in one-dimensional problems, we can apply accurate analytical solutions. Our work derives from that of Celia et al. [6] and Morton [30], while being tailored to the system of convection-reaction methods for arbitrary initial values. By transferring the problem to a mass transport we further conserve the underlying mass and have a conservative method, see [28].

For the larger scales, the relevant equations are diffusion and dispersion equations, and we use implicit temporal discretization. Higher-order methods are used by reconstruction with linear test exact test-functions. Therefore we obtain for the diffusion-dispersion equation implicit temporal discretization and the higher-order finite volume methods. The underlying linear system of equations is solved iteratively with a multi-grid solver. Our main advantage in this context is the coupling of the different equation types to obtain higher-order discretization methods.

Both results are coupled by the operator-splitting method, e.g. the Strang splitting method, to obtain at least second order in time and second order in space.

Using improved higher-order operator-splitting methods, we can improve our methods for the solution of the full equations.

Here we can use the knowledge of the analytical solutions of the convection-reaction part as test functions for the finite volume method. Compared with other methods, without using such analytical test function, e.g. WENO-methods (see $[22,23,26,33]$ ), we can also solve the reaction part exactly.

The methods are verified by benchmark problems and the numerical results are compared with the analytical solutions.

Here we apply test problems of waste disposals given in the literature [2,5]. For comparison we apply the analytical solutions which are given in one and two-dimensional benchmark problems.

A real-life problem is presented as a simulation of a waste disposal with realistic parameters and underlying layers in the porous media. The calculations are presented with figures and convergence results. Some applications containing this method are computed with the underlying program tool $R^{3} T$, and the main concepts are presented. A benchmark problem based on analytical solutions is introduced for testing the new discretization method and for presenting higher-order results.

The paper is comprised as follows. We introduce our mathematical model of a contaminant transport in flowing groundwater in Section 2. In Section 3 we introduce the finite volume methods to be used as basic discretization methods for our different equations. The modifications to the finite volume methods with respect to each equation type are explained in Section 4 for the convection part, in Section 5 for the reaction part, in Section 6 for the mixture of convection and reaction equations, and in Section 7 for the diffusion-dispersion part. The operator-splitting methods are presented in Section 8. Our numerical results with benchmark problems and realistic waste disposals are described in Section 9. Finally, we discuss our future works in Section 10 with respect to our research area.

\section{MAThematicAl MODEL}

We consider a steady-state groundwater flow that is described by a given velocity field $\boldsymbol{v}=\boldsymbol{v}(x)$ for $x \in \Omega \subset$ $\mathbb{R}^{d}$ for $d=2$ or $d=3$. In the groundwater several radionuclides (or some other chemical species) are dissolved.

We suppose that these nuclides take part in irreversible, first-order chemical reactions. In particular each nuclide (a "mother") can decay only to a single component (to a "daughter"), whereby each nuclide can be produced by several reactions, i.e. each daughter can have several mothers. 
Moreover, the radionuclides can be adsorbed to the soil matrix. If equilibrium linear sorption is assumed with different sorption constants for each component, the advective-dispersive transport of each component is slowed down by a different retardation factor.

To summarize, the mathematical model can be written in the form, see $[3,4,17]$,

$$
R^{(i)} \phi\left(\partial_{t} u^{(i)}+\lambda^{(i j)} u^{(i)}\right)+\nabla \cdot\left(\boldsymbol{v} u^{(i)}-D^{(i)} \nabla u^{(i)}\right)=\sum_{k} R^{(k)} \phi \lambda^{(k i)} u^{(k)},
$$

where $i=1, \ldots, I_{u}$. The integer $I_{u}$ denotes the total number of involved radionuclides. A stationary groundwater is supposed by considering only divergence-free velocity fields, i.e.

$$
\nabla \cdot \boldsymbol{v}(x)=0, \quad x \in \Omega
$$

The unknown functions $u^{(i)}=u^{(i)}(t, x)$ denote the concentrations of radionuclides, where the space and time variables $(t, x)$ are considered as $t \geq 0$ and $x \in \Omega$. The constant reaction rate $\lambda^{(i j)} \geq 0$ determines the decay (sink) term $\lambda^{(i j)} u^{(i)}$ for the concentration $u^{(i)}$ and the production (source) term for the concentration $u^{(j)}$. In general, the $j$ th radionuclide does not need to be included in the system (2.1), i.e. $j>I_{u}$. The indices $k$ on the right-hand side of (2.1) run through all mothers of the $i$ th radionuclide.

The remaining parameters in (2.1) include the diffusion-dispersion tensors $D^{(i)}=D^{(i)}(x, \boldsymbol{v})$, cf. [4], the retardation factors $R^{(i)}=R^{(i)}(x) \geq 1$, and the porosity of the medium $\phi=\phi(x)>0$.

In the following we concentrate on the modeling of processes on the boundary $\partial \Omega$ of the domain $\Omega$ and describe inflow and outflow boundaries.

We apply standard inflow and outflow boundary conditions. In particular, we neglect the diffusive-dispersive flux at the outflow (and "no-flow") boundary $\partial^{\text {out }} \Omega:=\{x \in \partial \Omega, \boldsymbol{n} \cdot \boldsymbol{v} \geq 0\}$,

$$
\boldsymbol{n} \cdot D^{(i)} \nabla u^{(i)}(t, \gamma)=0, \quad t>0, \quad \gamma \in \partial \Omega
$$

where $\boldsymbol{n}$ is the normal unit vector with respect to $\partial \Omega$. For the inflow boundary $\partial^{\text {in }} \Omega:=\{x \in \partial \Omega, \boldsymbol{n} \cdot \boldsymbol{v}<0\}$ we assume, that the concentrations are prescribed by Dirichlet boundary conditions

$$
u^{(i)}(t, \gamma)=U^{(i)}(t, \gamma), \quad t>0, \quad \gamma \in \partial^{\mathrm{in}} \Omega
$$

where the functions $U^{(i)}$ describe the boundary condition in the inflow boundary, see [19].

The initial conditions are considered in a general form,

$$
u^{(i)}(0, x)=U^{(i)}(0, x), \quad x \in \Omega
$$

Several authors present analytical solutions for the problem (2.1) on the assumption of a unidirectional constant velocity $\boldsymbol{v} \equiv(v, 0,0)$ and special boundary and initial conditions, see [19,37]. In this paper we introduce the finite volume discretization method to obtain a precise numerical solution of (2.1) for a general form of the velocity $\boldsymbol{v}, e . g . \boldsymbol{v}=\boldsymbol{v}(x)$, and for the general boundary and initial conditions (2.3)-(2.5).

The basic idea of our method is to apply a new second-order, explicit discretization scheme for the advectivereactive part of the system (2.1). This method is based on analytical solutions for locally one-dimensional problems on boundaries between two finite volumes, analogous to the well-known Godunov algorithm for purely advective problems, see [28]. This numerical method is locally mass conservative and it produces numerical solutions with no non-physical oscillations.

To solve the general model (2.1) numerically, we have to couple this new method with some standard discretizations for the dispersion part of (2.1) using the operator-splitting procedure. We present a standard "vertex-centered" finite volume method (or "control-volume finite element method", see [28]) for the discretization of the diffusive-dispersive part of the transport. 
Owing to the linearity of the equations in (2.1), we can split the problem (2.1) into several simpler problems. Applying afterwards the principle of superposition, we can obtain the solution of (2.1) by summing the solutions of such simpler problems.

These simpler systems are given for each $u^{(i)}$, where only a single linear decay chain with $u^{(i)}$ at the top is considered. As each nuclide decays only to a unique component of the system and only irreversible reactions are assumed, such decay chains are uniquely defined. Consequently, we end up with a problem in the form:

$$
R^{(i)} \phi\left(\partial_{t} u^{(i)}+\lambda^{(i)} u^{(i)}\right)+\nabla \cdot\left(\boldsymbol{v} u^{(i)}-D^{(i)} \nabla u^{(i)}\right)=R^{(i-1)} \phi \lambda^{(i-1)} u^{(i-1)},
$$

with the Dirichlet boundary conditions at the inflow boundary for $t>0$ and $\gamma \in \partial^{\text {in }} \Omega$

$$
\begin{array}{r}
u^{(1)}(t, \gamma)=U(t, \gamma), \\
u^{(i)}(t, \gamma)=0, \quad i=2, \ldots, I,
\end{array}
$$

and initial conditions for $x \in \Omega$,

$$
\begin{aligned}
u^{(1)}(0, x) & =U(0, x), \\
u^{(i)}(0, x)=0, \quad i & =2, \ldots, I .
\end{aligned}
$$

Clearly, the non-zero boundary and initial conditions $(2.4)-(2.5)$ are only considered for $u^{(i)}$, with all other components having zero initial concentrations and zero inflow concentrations. Moreover, for the first nuclide with concentration $u^{(1)}$ we formally define $\lambda^{(0)}=0$, i.e. the nuclide at the top only decays. Furthermore, all reaction constants $\lambda^{(i)}$, except the last one, are supposed to be strictly positive, i.e. $\lambda^{(i)}>0$ for $i=1,2, \ldots, I-1$, and $\lambda_{I} \geq 0$. Of course, $I \leq I_{u}$ holds.

In fact, the problem (2.6)-(2.10) can be further splitted into two simpler problems. First, non-zero initial conditions (2.10) are considered with zero concentration at the Dirichlet boundary, i.e. $U(t, \gamma) \equiv 0$ in $(2.7)$, and second, the zero initial conditions are taken, i.e. $U(0, x) \equiv 0$ in $(2.10)$, with general boundary conditions (2.7). Again, the sum of both solutions constitutes the solution of the problem (2.6)-(2.10).

Further on in this paper, we treat only the problem of the form (2.6)-(2.10).

\section{Finite volume methods}

The diffusion-dispersion part of the transport equation (2.6) will be discussed in Section 7.

To solve the remaining advection-reaction part of (2.6) by finite volume methods, we consider a mesh of nonempty non-intersecting finite volumes $\Omega_{j} \subset \Omega$ that cover $\Omega$. We assume that $\Omega_{j}, j=1, \ldots, N$ are polygonal (consequently, $\Omega$ must be polygonal, too).

To simplify the notation, we skip the index $i$ in $(2.6)$, i.e. $u:=u^{(i)}$, and we write:

$$
R \phi\left(\partial_{t} u+\lambda u\right)+\nabla \cdot(\boldsymbol{v} u)=Q
$$

where $Q=R^{(i-1)} \phi \lambda^{(i-1)} u^{(i-1)}$.

Integrating (3.1) over $\Omega_{j}$ and some time interval $\left(t^{n}, t^{n+1}\right)$, we obtain

$$
\int_{\Omega_{j}} R \phi u\left(t^{n+1}\right)=\int_{\Omega_{j}} R \phi u\left(t^{n}\right)-\int_{t^{n}}^{t^{n+1}} \int_{\partial \Omega_{j}} \boldsymbol{n}_{j} \cdot \boldsymbol{v} u+\int_{t^{n}}^{t^{n+1}} \int_{\Omega_{j}}(Q-\lambda u),
$$

where $\boldsymbol{n}_{j}$ is the unit normal vector with respect to the boundary $\partial \Omega_{j}$ of $\Omega_{j}$. For simplicity, we skip the integration variables in (3.2). 
Furthermore, we suppose that the porosity $\phi$ and the retardation factor $R$ have piecewise constant values that can be supposed physically and approximated to the finite volume mesh, i.e.

$$
\phi(x) \equiv \phi_{j}, \quad R(x) \equiv R_{j}, \quad x \in \Omega_{j} .
$$

For a general case of $\phi$ and $R$, which means we have to approximate the function to the finite volume mesh, we can apply averaging methods, see [8]. Note that $R_{j} \geq 1$.

Finally, we denote the averaged concentration $u_{j}^{n}$ of $u$ at $t=t^{n}$ in $\Omega_{j}$, i.e.

$$
u_{j}^{n}:=\frac{1}{\left|\Omega_{j}\right|} \int_{\Omega_{j}} u\left(t^{n}, x\right) \mathrm{d} x
$$

where $\left|\Omega_{j}\right|$ denotes the volume of $\Omega_{j}$. Analogously to (3.4), we can define the averaged values $u_{j}^{n+1}$.

Using the assumptions and notations from above, we can rewrite (3.2) in a discrete form,

$$
\left|\Omega_{j}\right| R_{j} \phi_{j} u_{j}^{n+1}=\left|\Omega_{j}\right| R_{j} \phi_{j} u_{j}^{n}-\sum_{k} \int_{t^{n}}^{t^{n+1}} \int_{\Gamma_{j k}} \boldsymbol{n}_{j} \cdot \boldsymbol{v} u+\int_{t^{n}}^{t^{n+1}} \int_{\Omega_{j}}(Q-\lambda u),
$$

where the index $k$ is considered only for neighbors $\Omega_{k}$ of $\Omega_{j}$ with common surfaces, i.e. $\Gamma_{j k}: \partial \Omega_{j} \cap \partial \Omega_{k}$. Note that the subscripts $j$ and $k$ here are related to the finite volume mesh, and that they should not be confused with superscripts in (2.1) reserved for indices of radionuclide components.

In fact, no numerical approximation was used in (3.5). Before applying a second-order numerical discretization for (3.5) with no time-splitting discretization error, we first discuss the solutions of the purely advective and purely reactive case of our model.

\section{Numerical solution of the ADVECtion EQUATION}

If no reactions are considered in (3.1), the remaining advection equation takes the following form:

$$
R \phi \partial_{t} u+\nabla \cdot(\boldsymbol{v} u)=0 .
$$

The initial conditions are given by (2.10), and $u(t, \gamma)$ is explicitly given for $t>0$ at the inflow boundary $\gamma \in \partial^{\text {in }} \Omega$ by $(2.7)$.

The exact solution of (4.1) can directly be defined by use of the so-called forward tracking form of characteristic curves. If the solution of (4.1) is known at some time point $t_{0} \geq 0$ and some point $y \in \Omega \cup \partial^{\text {in }} \Omega$, then $u$ remains constant for $t \geq t_{0}$ along the characteristic curve $X=X(t)$, i.e. $u(t, X(t))=u\left(t_{0}, y\right)$ and

$$
X(t)=X\left(t ; t_{0}, y\right)=y+\int_{t_{0}}^{t} \frac{\boldsymbol{v}(X(s))}{R(X(s)) \phi(X(s))} \mathrm{d} s .
$$

The characteristic curve $X(t)$ starts at the time $t=t_{0}$ in the point $y$, i.e. $X\left(t_{0} ; t_{0}, y\right)=y$, and it is tracked forward in time for $t>t_{0}$. Of course, we can obtain, that $X(t) \notin \Omega$, i.e. the characteristic curve can leave the domain $\Omega$ through $\partial^{\text {out } \Omega \text {. }}$

Consequently, we have that $u\left(t, X\left(t ; t_{0}, y\right)\right)=U\left(t_{0}, y\right)$, where the function $U(0, y)$ is given for $t_{0}=0$ and $y \in \Omega$ by initial conditions (2.10) and for $t_{0}>0$ and $y \in \partial^{\text {in }} \Omega$ by the inflow boundary conditions (2.7).

The solution $u(t, x)$ of $(4.1)$ can also be expressed in a "backward tracking" form, which is more suitable for a direct formulation of the discretization schemes. Concretely, for any characteristic curve $X=X(t)=X(t ; s, Y)$, that is defined in a forward manner, i.e. $X(s ; s, Y)=Y$ and $t \geq s$, we obtain the curve $Y=Y(s)=Y(s ; t, x)$, 


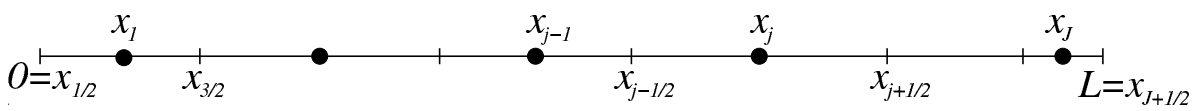

Figure 1. Notations for the one-dimensional case of the finite volume mesh.

that is defined in a backward manner, i.e. $Y(t ; t, X)=X$ and $s \leq t$. If we express $Y$ as function of $t_{0}$ for $t_{0} \leq t$, we obtain from (4.2):

$$
Y\left(t_{0}\right)=Y\left(t_{0} ; t, x\right)=x-\int_{t_{0}}^{t} \frac{\boldsymbol{v}(X(s))}{R(X(s)) \phi(X(s))} \mathrm{d} s,
$$

and we have $u(t, x)=u\left(t_{0}, Y\left(t_{0}\right)\right)$.

To simplify our treatment of inflow boundary conditions, we suppose that $U(t, \gamma)=U^{n+1 / 2} \equiv$ const. for $\gamma \in \partial^{\text {in }} \Omega$ and $t \in\left[t^{n}, t^{n+1}\right)$. Moreover, we define formally for any $\gamma \in \partial^{\text {in }} \Omega$ and $t_{0} \in\left[t^{n}, t^{n+1}\right]$, that $Y\left(s ; t_{0}, \gamma\right) \equiv$ $Y\left(t_{0} ; t_{0}, \gamma\right)$ for $t^{n} \leq s \leq t_{0}$.

In [15], the so-called flux-based (modified) method of characteristics was described. This method can be deemed as an extension of the standard finite volume methods (FVMs). From (3.5), the standard FVM for differential equations (4.1) takes the form:

$$
\left|\Omega_{j}\right| R_{j} \phi_{j} u_{j}^{n+1}=\left|\Omega_{j}\right| R_{j} \phi_{j} u_{j}^{n}-\sum_{k} \int_{t^{n}}^{t^{n+1}} \int_{\Gamma_{j k}} \boldsymbol{n}_{j}(\gamma) \cdot \boldsymbol{v}(\gamma) u(t, \gamma) \mathrm{d} \gamma \mathrm{d} t .
$$

The idea of a flux-based method of characteristics is to apply the substitution $u(t, \gamma)=u\left(t^{n}, Y\left(t^{n} ; t, \gamma\right)\right)$ on $(4.4)$.

Particularly, for the integration variable $t \in\left(t^{n}, t^{n+1}\right)$ and for each point $\gamma \in \partial^{\text {out }} \Omega_{j}$, the characteristic curves $Y(s)$ are tracked backward, starting in $\gamma$ at $s=t$ and ending in $s=t^{n}$. We must reach a point $Y=Y\left(t^{n}\right)$, such that $Y \in \partial^{\text {in }} \Omega$ or $Y \in \Omega$. In the first case, $u\left(t^{n}, Y\right)$ is given by the inflow concentration $U\left(t^{n}, Y\right)=U^{n}$, in the latter by $u\left(t^{n}, Y\right)$.

The integral on the right-hand side of (4.4) can be solved exactly for the one-dimensional case with general initial and boundary conditions, see [31]. For the general 2D or 3D case, a numerical approximation of $u\left(t_{0}, Y\left(t_{0}\right)\right)$, respectively of $Y\left(t_{0}\right)$, is to be used. First, we describe such an approximation in the one-dimensional case, and afterwards for the general $2 \mathrm{D}$ or $3 \mathrm{D}$ case.

\subsection{D case with a piecewise linear form of $u\left(t^{n}, x\right)$}

In the one-dimensional case the domain $\Omega \subset R$ is given by an interval $(0, L)$. Owing to $(2.2)$ we obtain that $v(x) \equiv v=$ const. and (4.1) takes the form:

$$
R \phi \partial_{t} u(t, x)+v \partial_{x} u(t, x)=0, \quad x \in(0, L), \quad t \geq 0
$$

Next, we treat the case $v>0$. An analogous treatment for a negative constant value of $v$ can easily be derived.

Let the finite volume mesh consist of $J+1$ intervals $\Omega_{j}:=\left(x_{j-1 / 2}, x_{j+1 / 2}\right)$ for $j=0,1, \ldots, J$, where we define $x_{-1 / 2} \equiv x_{0}=0$ and $x_{J+1 / 2} \equiv x_{J}=L$. The length of each interval is $h_{j}=x_{j+1 / 2}-x_{j-1 / 2}$, and the middle points $x_{j}$ for $j=1,2, \ldots, J-1$ are defined by $x_{j}:=x_{j-1 / 2}+h_{j} / 2$. For an illustration of the notation see Figure 1.

Let us now consider a particular form of the function $u$ at $t=t^{n}$ :

$$
u\left(t^{n}, x\right)=u_{j}^{n}+\sigma_{j}^{n}\left(x-x_{j}\right), \quad x \in\left(x_{j-1 / 2}, x_{j+1 / 2}\right] .
$$

This means that $u\left(t^{n}, x\right)$ is a piecewise linear function with respect to the finite volume mesh with some piecewise constant slopes (gradients) $\sigma_{j}^{n}$. For a general form of $u$ at $t=t^{n}$, we have to construct the function $u\left(t^{n}, x\right)$ 


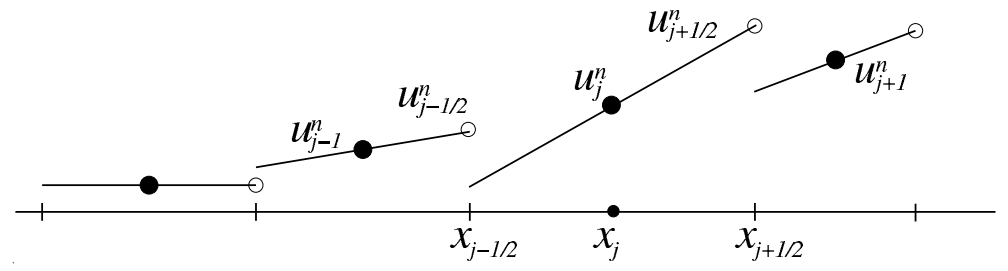

Figure 2. Piecewise linear form of $u\left(t^{n}, x\right)$.

of the form (4.6). For that, the values $u_{j}^{n}$ can be obtained with the averaging procedure (3.4), and the choices for the slopes $\sigma_{j}^{n}$ will be discussed in the next subsection.

Using (4.6), we can denote the value $u\left(t^{n}, x_{j+1 / 2}\right)$ at the outflow boundary point $x_{j+1 / 2}$ of $\Omega_{j}$ by

$$
u_{j+1 / 2}^{n}:=u_{j}^{n}+\sigma_{j}^{n} \frac{h_{j}}{2} .
$$

Note that $u\left(t^{n}, x\right)$ for $x \in(0, L)$ is generally discontinuous over the points $x_{j+1 / 2}$ (see Fig. 2 for an illustration).

The integral equation (4.4) can now be written in the one-dimensional form:

$$
h_{j} R_{j} \phi_{j} u_{j}^{n+1}=h_{j} R_{j} \phi_{j} u_{j}^{n}+v \int_{t^{n}}^{t^{n+1}}\left(u\left(t, x_{j-1 / 2}\right)-u\left(t, x_{j+1 / 2}\right)\right) \mathrm{d} t .
$$

We can easily show that for $v>0$ and some small time interval $t \in\left(t^{n}, t^{n+1}\right)$, we have

$$
\begin{aligned}
u\left(t, x_{j+1 / 2}\right) & =u\left(t^{n}, Y\left(t^{n} ; t, x_{j+1 / 2}\right)\right) \\
& =u_{j+1 / 2}^{n}+\left(u_{j}^{n}-u_{j+1 / 2}^{n}\right) \frac{2 v}{R_{j} \phi_{j} h_{j}}\left(t-t^{n}\right),
\end{aligned}
$$

and we have an analogous equation for $u\left(t, x_{j-1 / 2}\right)=u\left(t, x_{(j-1)+1 / 2}\right)$.

Clearly, the dependence on time of $u$ in (4.9) is linear and the integral in (4.8) can be computed exactly by means of the middle point quadrature rule. For that, we denote $u_{j+1 / 2}^{n+1 / 2}:=u\left(t^{n+1 / 2}, x_{j+1 / 2}\right)$ and obtain

$$
u_{j+1 / 2}^{n+1 / 2}=u_{j+1 / 2}^{n}+\frac{\tau^{n}}{\tau_{j}}\left(u_{j}^{n}-u_{j+1 / 2}^{n}\right),
$$

where $\tau_{j}$ is the so-called critical time-step, see [15]:

$$
\tau_{j}:=\frac{h_{j} R_{j} \phi_{j}}{v}
$$

Note that $\frac{\tau^{n}}{\tau_{j}}$ in (4.11) represents the so-called local grid Courant number.

The definitions (4.9)-(4.11) are valid, only if the time-step $\tau^{n}$ satisfies the so-called CFL (Courant-FriedrichsLewy) condition (see [28])

$$
\tau^{n} \leq \tau_{\mathrm{CFL}}:=\min \left\{\tau_{j}, j=0,1, \ldots, J\right\}
$$

The integral equation (4.8) can now be evaluated exactly for $j=0,1, \ldots, J$ by

$$
h_{j} R_{j} \phi_{j} u_{j}^{n+1}=h_{j} R_{j} \phi_{j} u_{j}^{n}+v \tau^{n}\left(u_{j-1 / 2}^{n+1 / 2}-u_{j+1 / 2}^{n+1 / 2}\right),
$$

where $u_{-1 / 2}^{n+1 / 2} \equiv U^{n+1 / 2}$. 


\subsection{Construction of the piecewise linear form of $u\left(t^{n}, x\right)$}

To finalize the discretization scheme (4.14), we have to choose (or construct) the slopes $\sigma_{j}^{n}$. It is well-known (see [28]) that $\sigma_{j}^{n}$ must be chosen carefully, otherwise non-physical oscillations can be observed in the numerical solution of (4.14). These can include under- and overshootings of physically acceptable values for the solution.

To formulate precisely these difficulties and their solutions, we introduce the concept of flux limiters (or slope limiters) based on the local discrete minimum and maximum principle for numerical solutions of (4.14), as introduced in [15].

The simplest choice for the slopes $\sigma_{j}^{n}$ in (4.6) is to set them all equal to zero, so that we obtain $u_{j+1 / 2}^{n} \equiv u_{j}^{n}$. Consequently, the discretization scheme (4.14) turns into the well-known first-order upwind scheme

$$
h_{j} R_{j} \phi_{j} u_{j}^{n+1}=h_{j} R_{j} \phi_{j} u_{j}^{n}+v \tau^{n}\left(u_{j-1}^{n}-u_{j}^{n}\right) .
$$

For equations (4.15), the local discrete minimum and maximum principle is satisfied for $j=1, \ldots, J$,

$$
\min \left\{u_{j}^{n}, u_{j-1}^{n}\right\}=: u_{j, \min }^{n} \leq u_{j}^{n+1} \leq u_{j, \max }^{n}:=\max \left\{u_{j}^{n}, u_{j-1}^{n}\right\},
$$

if the CFL condition (4.13) is valid.

The property (4.16) is a consequence of the circumstance that for (4.15) the trivial solution $u_{j}^{n+1}=u_{j}^{n}=$ $u_{j-1}^{n}=1$ is valid, that the coefficient before $u_{j}^{n+1}$ in (4.15) is positive, i.e. $h_{j} R_{j} \phi_{j}>0$, the coefficients before $u_{j}^{n}$ and $u_{j-1}^{n}$ are non-negative, i.e. $h_{j} R_{j} \phi_{j}-v \tau^{n} \geq 0$, and $v \tau^{n}>0$.

To propose a higher-resolution form of (4.14), we can follow the approach of [28] to reconstruct the piecewise linear form of $u\left(t^{n}, x\right)$ in (4.6) by defining the slopes $\sigma_{j}^{n}$ with the values $u_{j-1}^{n}, u_{j}^{n}$ and $u_{j+1}^{n}$.

Here we mention only two particular choices of $\sigma_{j}^{n}$ : for other forms and more detailed discussion, see [28]. First, as $\sigma_{j}^{n}$ is used to define the value $u_{j+1 / 2}^{n}$ in (4.11), we can naturally construct the slope $\sigma_{j}^{n}$ with only two values $u_{j}^{n}$ and $u_{j+1}^{n}$,

$$
\sigma_{j}:=\frac{u_{j+1}-u_{j}}{x_{j+1}-x_{j}}
$$

or, to consider all three values $u_{j-1}^{n}, u_{j}^{n}$ and $u_{j+1}^{n}$, we can use the averaged gradient over $\left(x_{j-1}, x_{j+1}\right)$,

$$
\sigma_{j}:=\frac{u_{j+1}-u_{j-1}}{x_{j+1}-x_{j-1}}=\frac{x_{j+1}-x_{j}}{x_{j+1}-x_{j-1}} \frac{u_{j+1}-u_{j}}{x_{j+1}-x_{j}}+\frac{x_{j}-x_{j-1}}{x_{j+1}-x_{j-1}} \frac{u_{j}-u_{j-1}}{x_{j}-x_{j-1}}
$$

and the choice (4.17) leads to the so-called Lax-Wendroff method with

$$
u_{j+1 / 2}^{n}=\frac{1}{2}\left(u_{j}^{n}+u_{j+1}^{n}\right),
$$

the choice (4.18) leads to the so-called Fromm method, see [28]. A generalization of these two methods for the $2 \mathrm{D}$ and $3 \mathrm{D}$ case using unstructured grids will be presented in the next subsection.

We now formulate the conditions, that lead to the local discrete minimum and maximum principle for (4.14) with (4.17) or (4.18).

After substitution of (4.11) into (4.14), we obtain

$$
h_{j} R_{j} \phi_{j} u_{j}^{n+1}=\left(h_{j} R_{j} \phi_{j}-v \tau^{n} \frac{\tau^{n}}{\tau_{j}}\right) u_{j}^{n}-v \tau^{n}\left(1-\frac{\tau^{n}}{\tau_{j}}\right) u_{j+1 / 2}^{n}+v \tau^{n} u_{j-1 / 2}^{n+1 / 2} .
$$

The coefficient before $u_{j+1 / 2}^{n}$ in (4.20) is negative, and the discrete min-max principle (4.16) can not be directly obtained. Nevertheless, we can rewrite (4.20) into a form where all coefficients are non-negative by introducing the values $u_{1 / 2+j}^{n}$ (using a rather formal notation):

$$
u_{1 / 2+j}^{n}:=2 u_{j}^{n}-u_{j+1 / 2}^{n} \quad \Rightarrow \quad u_{j+1 / 2}^{n}=2 u_{j}^{n}-u_{1 / 2+j}^{n} .
$$


By substituting (4.11) and (4.21) to (4.14), we obtain

$$
h_{j} R_{j} \phi_{j} u_{j}^{n+1}=\left(h_{j} R_{j} \phi_{j}-v \tau^{n}\left(2-\frac{\tau^{n}}{\tau_{j}}\right)\right) u_{j}^{n}+v \tau^{n}\left(1-\frac{\tau^{n}}{\tau_{j}}\right) u_{1 / 2+j}^{n}+v \tau^{n} u_{j-1 / 2}^{n+1 / 2} .
$$

Now we can easily show that all coefficients in (4.22) are non-negative, if the CFL condition (4.13) is satisfied, because of

$$
h_{j} R_{j} \phi_{j}-v \tau^{n}\left(2-\frac{\tau^{n}}{\tau_{j}}\right)=v\left(\tau_{j}-\tau^{n}\right)\left(1-\frac{\tau^{n}}{\tau_{j}}\right) .
$$

Consequently, the local discrete min-max principle can be formulated with respect to the values of $u$ on the right-hand side of (4.22). Of course, we want to obtain the local discrete min-max principle with respect to the values $u_{j \text {,min }}^{n}$ and $u_{j, \max }^{n}$ defined in (4.16). For that the two following conditions must be fulfilled:

$$
\begin{aligned}
& u_{j, \text { min }}^{n} \leq u_{1 / 2+j}^{n} \leq u_{j, \text { max }}^{n}, \\
& u_{j, \min }^{n} \leq u_{j-1 / 2}^{n+1 / 2} \leq u_{j, \max }^{n} .
\end{aligned}
$$

In general the conditions (4.23)-(4.24) are not fulfilled and we have to apply the so-called limiters to avoid non-physical oscillations in the numerical solution of (4.14), as well as the so-called phase error [28].

Using the discrete min-max principle and the equivalent formulation (4.22) to (4.14), it is very easy and straightforward to formulate such limiters. The idea is to replace the value $u_{1 / 2+j}^{n}$ in (4.22) in the case of $u_{1 / 2+j}^{n} \notin$ $\left[u_{j, \min }^{n}, u_{j, \max }^{n}\right]$ by the corresponding extreme value that was violated $\left(u_{j-1 / 2}^{n+1 / 2}\right.$ can be replaced analogously).

In particular, the discretization scheme (4.14) can be replaced by a flux-limited version

$$
h_{j} R_{j} u_{j}^{n+1}=h_{j} R_{j} u_{j}^{n}+v \tau^{n}\left(\tilde{u}_{j-1 / 2}^{n+1 / 2}-\tilde{u}_{j+1 / 2}^{n+1 / 2}\right)
$$

where

$$
\tilde{u}_{j+1 / 2}^{n+1 / 2}:=\left\{\begin{array}{lll}
u_{j+1, \max }^{n} & & \bar{u}_{j+1 / 2}^{n+1 / 2}>u_{j+1, \max }^{n} \\
\bar{u}_{j+1 / 2}^{n+1 / 2} & u_{j+1, \min }^{n} \leq \bar{u}_{j+1 / 2}^{n+1 / 2} \leq u_{j+1, \max }^{n} \\
u_{j+1, \min }^{n} & u_{j+1, \min }^{n}>\bar{u}_{j+1 / 2}^{n+1 / 2},
\end{array}\right.
$$

and

$$
\bar{u}_{j+1 / 2}^{n+1 / 2}=\bar{u}_{j+1 / 2}^{n}+\frac{\tau^{n}}{\tau_{i}}\left(u_{j}^{n}-\bar{u}_{j+1 / 2}^{n}\right),
$$

with

$$
\bar{u}_{j+1 / 2}^{n}:=\left\{\begin{array}{lr}
2 u_{j}^{n}-u_{j, \max }^{n} & 2 u_{j}^{n}-u_{j+1 / 2}^{n+1 / 2}>u_{j, \max }^{n} \\
u_{j+1 / 2}^{n} & u_{j, \min }^{n} \leq 2 u_{j}^{n}-u_{j+1 / 2}^{n+1 / 2} \leq u_{j, \max }^{n} \\
2 u_{j}^{n}-u_{j, \min }^{n} & u_{j, \min }^{n}>2 u_{j}^{n}-u_{j+1 / 2}^{n+1 / 2} .
\end{array}\right.
$$

The last limiter (4.28) is necessary to fulfill (4.23) for the $j$ th discrete equation (4.14).

The first limiter (4.26) is necessary to fulfill (4.24) for the $(j+1)$ st discrete equation (4.14)). It does not need to be used for the Lax-Wendroff discretization scheme (4.17), where we can directly use $\tilde{u}_{j+1 / 2}^{n+1 / 2} \equiv \bar{u}_{j+1 / 2}^{n+1 / 2}$.

The limiting procedure $(4.26)-(4.28)$ cannot fail, because the most limiting choice $\tilde{u}_{j+1 / 2}^{n+1 / 2}=u_{j}^{n}$, i.e. $\tilde{\sigma}_{j}^{n}=0$, is always available.

The advantage of the high-resolution finite volume scheme (4.25) is that it can be straightforwardly applied to $2 \mathrm{D} / 3 \mathrm{D}$ advection equations computed on unstructured grids (see the next section). 


\section{3. $2 \mathrm{D} / 3 \mathrm{D}$ case}

It is quit straightforward to extend the discretization scheme (4.25) for a $2 \mathrm{D} / 3 \mathrm{D}$ case of the advection equation (4.1). To do this, we first denote

$$
v_{j k}:=\int_{\Gamma_{j k}} \boldsymbol{n}_{j}(\gamma) \cdot \boldsymbol{v}(\gamma) \mathrm{d} \gamma
$$

Of course, $v_{k j}=-v_{j k}$. Further, we call a boundary $\Gamma_{j k}$ of $\partial \Omega_{j}$ to be the "outflow" boundary, if $v_{j k}>0$, and, analogously, "inflow" boundary, if $v_{j k} \leq 0$. Similarly, we denote $k \in \operatorname{out}(j)$, if $v_{j k}>0$, and, consequently, $j \in \operatorname{in}(k)$.

Owing to $\nabla \cdot \boldsymbol{v}$, we have

$$
\sum_{k \in \operatorname{in}(j)} v_{k j}=\sum_{k \in \operatorname{out}(j)} v_{j k}
$$

We can regard (4.30) as the mass conservation property for the groundwater flow in a discrete form.

In fact, a numerical approximation of $v_{j k}$ in (4.29) can be used if we choose the middle point $\gamma_{j k}$ of $\Gamma_{j k}$ and

$$
v_{j k}: \approx\left|\Gamma_{j k}\right| \boldsymbol{n}_{j}\left(\gamma_{j k}\right) \cdot \boldsymbol{v}\left(\gamma_{j k}\right)
$$

where $\left|\Gamma_{j k}\right|$ denotes the measure of $\Gamma_{j k}$. Consequently, the discrete form (4.30) of the mass balance property does not need to be exactly fulfilled in such a case, but very often the groundwater velocity field is given as a result of the numerical modeling, where the discrete equations (4.30) were included, and hence we suppose in all following considerations that (4.30) is valid.

Using the notations and approximations from above, we can approximate (4.4) by

$$
\left|\Omega_{j}\right| R_{j} \phi_{j} u_{j}^{n+1}=\left|\Omega_{j}\right| R_{j} \phi_{j} u_{j}^{n}-\sum_{k} v_{j k} \int_{t^{n}}^{t^{n+1}} u\left(t^{n}, Y\left(t^{n} ; t, \gamma_{j k}\right)\right) \mathrm{d} t .
$$

The simplest discretization scheme for (4.32) is to assume a piecewise constant form of $u\left(t^{n}, x\right)$ with respect to the finite volume mesh, which means $u\left(t^{n}, x\right)=u_{j}^{n}$ for $x \in \Omega_{j}$. Consequently, for "enough small" time-steps $\tau^{n}$, the characteristic curves $Y\left(t^{n} ; t, \gamma_{j k}\right)$, that start at outflow boundaries $\gamma_{j k} \in \Gamma_{j k}$ remain for $t \in\left(t^{n}, t^{n}+\tau^{n}\right)$ in $\Omega_{j}$ and thus $u\left(t^{n}, Y\left(t^{n} ; t, \gamma_{j k}\right)\right) \equiv u_{j}^{n}$.

Using the assumptions from above, we can evaluate (4.32) by

$$
\left|\Omega_{j}\right| R_{j} \phi_{j} u_{j}^{n+1}=\left|\Omega_{j}\right| R_{j} \phi_{j} u_{j}^{n}-\tau^{n} u_{j}^{n} \sum_{k \in \operatorname{out}(j)} v_{j k}+\tau^{n} \sum_{k \in \operatorname{in}(j)} v_{k j} u_{k}^{n} .
$$

The discretization scheme (4.33) can be regarded as the first-order upwind (upstreaming) scheme. If the time-step is restricted by the CFL condition $\tau^{n} \leq \tau_{C F L}$ (see (4.13)), then the numerical solution of (4.33) satisfies the local discrete minimum and maximum principle

$$
u_{j, \min }^{n} \leq u_{i}^{n+1} \leq u_{j, \max }^{n}
$$

where the extreme values are given by

$$
u_{j, \text { min }}^{n}:=\min \left\{u_{j}^{n} ; u_{k}^{n}, k \in \operatorname{in}(j)\right\}, \quad u_{j, \max }^{n}:=\max \left\{u_{j}^{n} ; u_{k}^{n}, k \in \operatorname{in}(j)\right\} .
$$

The critical time-steps $\tau_{i}$ in (4.13) are now defined by

$$
\tau_{i}:=\frac{\left|\Omega_{j}\right| R_{j} \phi_{j}}{v_{j}}
$$


where $v_{j}$ denotes the total outflow (or inflow) flux for $\Omega_{j}$, i.e.

$$
v_{j}:=\sum_{k \in \operatorname{out}(j)} v_{j k}
$$

The extension of the one-dimensional high-resolution scheme (4.14) on the 2D or 3D case can be derived in several different ways, obtaining at the end several different discretization schemes. Here we present the simplest and the most straightforward one,

$$
\left|\Omega_{j}\right| R_{j} \phi_{j} u_{j}^{n+1}=\left|\Omega_{j}\right| R_{j} \phi_{j} u_{j}^{n}-\tau^{n} \sum_{k \in \operatorname{out}(j)} v_{j k} u_{j k}^{n+1 / 2}+\tau^{n} \sum_{k \in \operatorname{in}(j)} v_{k j} u_{k j}^{n+1 / 2},
$$

where

$$
u_{j k}^{n+1 / 2}:=u_{j k}^{n}+\frac{\tau^{n}}{\tau_{i}}\left(u_{j}^{n}-u_{j k}^{n}\right) .
$$

The value $u_{j k}^{n}$ should represent (approximately) the value of $u$ at $x_{i j}:=0.5\left(x_{i}+x_{j}\right)$. The simplest choice

$$
u_{j k}^{n}:=\frac{1}{2}\left(u_{j}^{n}+u_{k}^{n}\right)
$$

can be regarded as the generalization of the Lax-Wendroff scheme (4.19). A general choice can be derived from

$$
u_{j k}^{n}:=u_{j}^{n}+\sigma_{j k}^{n} \cdot\left(x_{j k}-x_{j}\right)
$$

where $\boldsymbol{\sigma}_{j k}^{n}$ is a reconstructed gradient used for the determination of $u_{j k}^{n}$. Later we describe a particular algorithm for the reconstruction of $\boldsymbol{\sigma}_{j k}^{n} \equiv \boldsymbol{\sigma}_{j}^{n}$ for the so-called vertex-centered finite volumes.

Theorem 4.1. We have a second-order method for the equation (4.40), and our approximation error is given as:

$$
\left|u\left(t^{n}, x_{j k}\right)-u_{j k}^{n}\right| \leq O\left(\left(\Delta x_{j k}\right)^{2}\right)
$$

where $u\left(x_{j k}, t^{n}\right)$ is the approximated solution and $u_{j k}^{n}$ is the exact solution. Further $\Delta x_{j k}=\left(x_{j k}-x_{j}\right)$ is the spatial step size of the underlying grid.

Proof. Using the literature for conservation laws (see $[27,28]$ ), we start with the reconstruction of the approximate solution $u_{j k}^{n}$ given as

$$
u_{j k}^{n}:=u_{j}^{n}+\boldsymbol{\sigma}_{j k}^{n} \cdot\left(x_{j k}-x_{j}\right),
$$

where $\boldsymbol{\sigma}_{j k}^{n}$ is a reconstructed gradient used for the determination of $u_{j k}^{n}$.

If we reset $\boldsymbol{\sigma}_{j k}^{n}$ with a reconstructed gradient $\nabla u_{j k}^{n}$, we obtain

$$
u_{j k}^{n}:=u_{j}^{n}+\nabla u_{j k}^{n} \cdot\left(x_{j k}-x_{j}\right),
$$

where $\nabla u_{j k}^{n}$ is reconstructed with the neighborhood elements (see $[1,34]$ ).

Furthermore, our analytical solution $u\left(x_{j k}, t^{n}\right)$ is given as:

$$
u\left(t^{n}, x_{j k}\right):=u_{j}^{n}+\nabla u_{j k}^{n} \cdot\left(x_{j k}-x_{j}\right)+\Delta u_{j k}^{n} \cdot\left(x_{j k}-x_{j}\right)^{2}+O\left(\left(x_{j k}-x_{j}\right)^{3}\right) .
$$

By subtracting the analytical solution (4.44) of the approximate solution (4.43), we obtain the following error,

$$
\left|u\left(t^{n}, x_{j k}\right)-u_{j k}^{n}\right| \leq O\left(\left(x_{j k}-x_{j}\right)^{2}\right)
$$

where we obtain a second-order accurate method (see also $[28,38]$ ). This proves our statement of the theorem. 
For methods higher than second-order we have to approximate the second derivative of the Taylor expansion (see also [34]). It may be more delicate because of further reconstructions and limiting processes with the neighbor and neighbor-neighbor elements.

In the next step we prove the discrete min-max principle for (4.37). We rewrite it formally into the form

$$
\begin{aligned}
\left|\Omega_{j}\right| R_{j} \phi u_{j}^{n+1}= & \left(\left|\Omega_{j}\right| R_{j} \phi_{j}-\tau^{n} v_{j}\left(2-\frac{\tau^{n}}{\tau_{i}}\right)\right) u_{j}^{n}+ \\
& \tau^{n} \sum_{k \in \operatorname{out}(j)} v_{j k}\left(1-\frac{\tau^{n}}{\tau_{i}}\right) u_{j k^{\prime}}^{n}+\tau^{n} \sum_{k \in \operatorname{in}(j)} v_{k j} u_{k j}^{n+1 / 2},
\end{aligned}
$$

where the value $u_{j k^{\prime}}^{n}$ is given analogously to (4.21) by

$$
u_{j k}^{n}=2 u_{j}^{n}-u_{j k^{\prime}}^{n}
$$

Based on (4.46), we formulate the final discretization scheme with the limiter

$$
\left|\Omega_{j}\right| R_{j} u_{j}^{n+1}=\left|\Omega_{j}\right| R_{j} u_{j}^{n}-\tau^{n} \sum_{k \in \operatorname{out}(j)} v_{j k} \tilde{u}_{j k}^{n+1 / 2}+\tau^{n} \sum_{k \in \operatorname{in}(j)} v_{k j} \tilde{u}_{k j}^{n+1 / 2},
$$

where

and

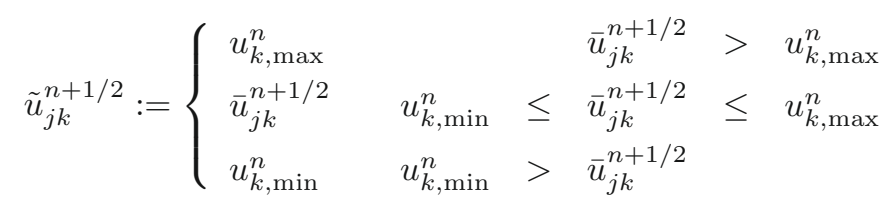

$$
\bar{u}_{j k}^{n+1 / 2}:=\bar{u}_{j k}^{n}+\frac{\tau^{n}}{\tau_{i}}\left(u_{j}^{n}-\bar{u}_{j k}^{n}\right)
$$

where

$$
\bar{u}_{j k}^{n}:=\left\{\begin{array}{lr}
2 u_{j}^{n}-u_{j, \max }^{n} & 2 u_{j}^{n}-u_{j k}^{n}>u_{j, \max }^{n} \\
u_{j k}^{n} & u_{j, \min }^{n} \leq 2 u_{j}^{n}-u_{j k}^{n} \leq u_{j, \max }^{n} \\
2 u_{j}^{n}-u_{j, \min }^{n} & u_{j, \min }^{n}>2 u_{j}^{n}-u_{j k}^{n} .
\end{array}\right.
$$

With this approach, the so-called grid effect can corrupt the obtained numerical solutions and we have satisfied the discrete min-max principle. At least the result of the improved approximation is based on the middle-point rule, which preserves the min-max principle.

The next section describes the analytical solutions of the reaction-equation system.

\section{Solution of the Reaction equation System}

If we consider only the reaction part of the general equation (2.6), we have a system of ordinary differential equations,

$$
\begin{aligned}
& R^{(1)} \phi\left(\partial_{t} u^{(1)}+\lambda^{(1)} u^{(1)}\right)=0, \quad u^{(1)}(x)=U(0, x), \\
& R^{(i)} \phi\left(\partial_{t} u^{(i)}+\lambda^{(i)} u^{(i)}\right)=R^{(i-1)} \phi \lambda^{(i-1)} u^{(i-1)}, \quad u^{(i)}(x)=0,
\end{aligned}
$$

for $i=2, \ldots, I$, where the dependence on the space variable $x \in \Omega$ is realized only through initial conditions for the first component and through retardation factors $R^{(i)}$. 
Theorem 5.1. For different reaction constants $\lambda^{(i)}$, we can derive an analytical solution of equations (5.1)-(5.2). Our exact solution is given by

$$
u^{(i)}(t, x)=U(0, x) \frac{R^{(1)}(x)}{R^{(i)}(x)} \Lambda_{i} \sum_{j=1}^{i} \Lambda_{j, i} \exp \left(-\lambda^{(j)} t\right),
$$

where the constants $\Lambda_{i}$ and $\Lambda_{j, i}$ for $j=1, \ldots, i$ are defined as:

$$
\begin{array}{r}
\Lambda_{1}:=1, \quad \Lambda_{i}:=\prod_{k=1}^{i-1} \lambda^{(k)}, \quad i=2, \ldots, I, \\
\Lambda_{1,1}:=1, \quad \Lambda_{j, i}:=\prod_{\substack{k=1 \\
k \neq j}}^{i} \frac{1}{\lambda^{(k)}-\lambda^{(j)}}, \quad i=2, \ldots, I .
\end{array}
$$

Proof. The analytical solution is derived by the Laplacian transformation, see $[7,18]$.

The exact solution of the reaction equations can be used for the numerical solving of the general system (2.6) using the so-called operator-splitting method (see, e.g. [28]).

\section{General advection-Reaction discretization scheme}

The system of equations for the convective transport with decay reactions takes the form:

$$
\partial_{t}\left(R^{(l)} \phi u^{(l)}\right)+\nabla \cdot\left(\boldsymbol{v} u^{(l)}\right)+\lambda^{(l)} R^{(l)} \phi u^{(l)}=\lambda^{(l-1)} R^{(l-1)} \phi u^{(l-1)} .
$$

Usually, we can use the exact scheme (5.3) for the decay part of (6.1) and the explicit discretization scheme (4.37) for the transport part. If the parameters $\theta^{(l)}$ for each component are not very different, i.e. $\theta^{(l)} \approx \theta$ for $l=1, \ldots, I$, then the operator-splitting approach (see [28]) only has a small temporal splitting error, and it can be successfully used for (6.1).

In general, however, e.g. if the retardation factors are different, the convection and the reaction operator do not commute, and the standard operator-splitting method can have a large temporal splitting error.

Here we present a novel algorithm for the computations of (6.1).

Concretely, for each $\Omega_{i}$ and $j \in \operatorname{out}(i)$, we have the following system of $1 \mathrm{D}$ convection-reaction equations for $l=1, \ldots, I$ :

$$
R_{i}^{(l)} \phi_{i} \partial_{t} u_{i}^{(l)}+v_{i j} \partial_{x} u_{i}^{(l)}+\lambda^{(l)} R_{i}^{(l)} \phi_{i} u_{i}^{(l)}=\lambda^{(l-1)} R_{i}^{(l-1)} \phi_{i} u_{i}^{(l-1)} .
$$

The equation can be solved exactly for particular initial conditions, and this is discussed in the next Section 6.1. In the following, we consider the space variable $x \in(0, \infty)$, and the velocity $v_{i j}>0$, given by (4.29).

We rewrite the concentration $c_{i}^{(l)}:=R_{i}^{(l)} \phi_{i} u_{i}^{(l)}$ and the velocity $\tilde{v}_{i j}:=\frac{v_{i j}}{R_{i}^{(l)} \phi_{i}}$ and obtain:

$$
\partial_{t} c_{i}^{(l)}+\tilde{v}_{i j} \partial_{x} c_{i}^{(l)}+\lambda^{(l)} c_{i}^{(l)}=\lambda^{(l-1)} c_{i}^{(l-1)} .
$$

Because of the time restriction, we first calculate the maximal time-step for the cell $j$ and the concentration $i$ with respect to the total outflow fluxes,

$$
\tau_{l, i}=\frac{V_{i} R^{(l)}}{\nu_{i}}, \quad \nu_{i}=v_{i j}, j=\operatorname{out}(i) .
$$


We get the restricted time-step with the local time-step of each of the cells and their underlying components

$$
\tau^{n} \leq \min _{\substack{l=1, \ldots, m \\ i=1, \ldots, I}} \tau_{l, i}
$$

The velocity of the discrete equation is given as

$$
v_{l, i}=\frac{1}{\tau_{l, i}}
$$

We calculate the analytical solution of the mass (see the next Sect. 6.1), by using equations (6.17) and (6.18). We obtain

$$
\begin{gathered}
m_{i j, \text { rest }}^{(l), n}=m_{1}^{(l), n}\left(a, b, \tau^{n}, v_{1, i}, \ldots, v_{l, i}, R^{(1)}, \ldots, R^{(l)}, \lambda^{(1)}, \ldots, \lambda^{(l)}\right) \\
m_{i j, \text { out }}^{(l), n}=m_{2}^{(l)}\left(a, b, \tau^{n}, v_{1, i}, \ldots, v_{l, i}, R^{(1)}, \ldots, R^{(l)}, \lambda^{(1)}, \ldots, \lambda^{(l)}\right)
\end{gathered}
$$

where $a=V_{i} R^{(l)}\left(c_{i j}^{(l), n}-c_{i j^{\prime}}^{(l), n}\right), b=V_{i} R^{(l)} c_{i j^{\prime}}^{(l), n}$ and $m_{i}^{(l), n}=V_{i} R^{(l)} c_{i}^{(l), n}$ are the parameters and $j=\operatorname{out}(i)$, $j^{\prime}=\operatorname{in}(i)$ are the indices of the flows. The linear impulse in the finite volume cell is constructed by $c_{i j^{\prime}}^{(l), n}$ for the concentration on the inflow boundary and $c_{i j}^{(l), n}$ for the concentration on the outflow boundary of the cell $i$.

The discretization with the embedded analytical mass is calculated by

$$
m_{i}^{(l), n+1}=m_{i j, \text { rest }}^{(l), n}+m_{j^{\prime} i, \text { out }}^{(l), n}
$$

where $m_{i j, \text { rest }}^{(l), n}=m_{i}^{(l), n}-m_{i j, \text { out }}^{(l), n}$ is the residual mass coming from the total mass and the outflown mass. The mass in the next time-step is $m_{i}^{(l), n+1}=V_{i} c_{i}^{(l), n+1}$, and in the old time-step it is the residual mass for the concentration $l$. The proof is shown in [18]. In the next section we derive an analytical solution for the benchmark problem.

\subsection{Exact mass solutions for 1D system of advection-reaction equations}

For a simpler computation, we transform the cell $\Omega_{i}=(0, L)$ to a unit cell $\Omega_{i}=(0,1)$. We also have to arrange $c^{(l)}=R^{(l)} \phi u^{(l)}$ to obtain a simpler system of equations. For the one-dimensional mass solution simpler equations can be derived.

The analytical solution for the remaining mass in the unit cell is given in the following Theorem 6.1.

Theorem 6.1. For the one-dimensional equation (6.3), we can compute the remaining mass of the unit cell as

$$
\begin{aligned}
m_{1}^{(l)}=\Lambda^{(l)} & \sum_{i=1}^{l} \Lambda_{i}^{(l)} \exp \left(-\lambda_{i} t\right)\left(a \frac{\left(1-v_{i} t\right)^{2}}{2}+b\left(1-v_{i} t-\sum_{\substack{j=1 \\
j \neq i}}^{l} \frac{1}{\lambda_{i j}}\right)\right. \\
& \left.-a\left(1-v_{i} t\right)\left(\sum_{\substack{j=1 \\
j \neq i}}^{l} \frac{1}{\lambda_{i j}}\right)+a\left(\sum_{\substack{j=1 \\
j \neq i}}^{l} \frac{1}{\lambda_{i j}}\left(\sum_{\substack{k \geq j \\
k \neq i}}^{l} \frac{1}{\lambda_{i k}}\right)\right)\right)
\end{aligned}
$$

where the factors $\lambda_{i j}$ are defined as:

$$
\lambda_{j i}=\lambda_{i j}:=\frac{\lambda^{(i)}-\lambda^{(j)}}{v^{(i)}-v^{(j)}},
$$


and the factors $\Lambda^{(l)}, \Lambda_{j}^{(l)}$ and $\Lambda_{i j}^{(l)}$ are given as:

$$
\Lambda^{(l)}=\prod_{i=1}^{l-1} \lambda_{i}, \Lambda_{i}^{(l)}=\left(\prod_{\substack{i=1 \\ j \neq i}}^{l} \frac{1}{\lambda_{j}-\lambda_{i}}\right), \Lambda_{i j}^{(l)}=\left(\prod_{\substack{k=1 \\ k \neq j \\ k \neq i}}^{l} \frac{\lambda_{i k}}{\lambda_{i k}-\lambda_{i j}}\right)
$$

Proof. The idea is to subdivide equation (6.4) into four subequations, and these are ordered according to the factors $1, \frac{1}{\lambda_{j k}}, \frac{1}{\lambda_{j k}^{2}}$, and $\exp (\cdot)$. After rearrangement the following four subequations appear:

$$
\begin{aligned}
& m_{1}^{(l)}=m_{1_{1}}^{(l)}+m_{1_{2}}^{(l)}+m_{1_{3}}^{(l)}+m_{1_{4}}^{(l)}, \\
& m_{1_{1}}^{(l)}=\Lambda^{(l)}\left(\sum_{j=1}^{l} \exp \left(-\lambda_{j} t\right) \Lambda_{j}^{(l)} \sum_{\substack{k=1 \\
k \neq j}}^{l} \Lambda_{j k}^{(l)}\left(a \frac{\left(1-v_{j} t\right)^{2}}{2}+b\left(1-v_{j} t\right)\right)\right), \\
& m_{1_{2}}^{(l)}=\Lambda^{(l)}\left(\sum_{j=1}^{l} \exp \left(-\lambda_{j} t\right) \Lambda_{j}^{(l)} \sum_{\substack{k=1 \\
k \neq j}}^{l} \Lambda_{j k}^{(l)}\left(-a \frac{1}{\lambda_{j k}}\left(1-v_{j} t\right)-b \frac{1}{\lambda_{j k}}\right)\right), \\
& m_{1_{3}}^{(l)}=\Lambda^{(l)}\left(\sum_{j=1}^{i} \exp \left(-\lambda_{j} t\right) \Lambda_{j}^{(l)} \sum_{\substack{k=1 \\
k \neq j}}^{l} \Lambda_{j k}^{(l)} \frac{a}{\lambda_{j k}^{2}}\right), \\
& m_{1_{4}}^{(l)}=\Lambda^{(l)}\left(\sum_{j=1}^{i} \exp \left(-\lambda_{j} t\right) \Lambda_{j}^{(l)} \sum_{\substack{k=1 \\
k \neq j}}^{l} \Lambda_{j k}^{(l)}\left(b-\frac{a}{\lambda_{j k}}\right) \frac{1}{\lambda_{j k}} \exp \left(-\lambda_{j k}\left(1-v_{j} t\right)\right)\right) .
\end{aligned}
$$

Subsequently, equation (6.11) is simplified by remodeling into symmetric terms, that pairwise cancel themselves out.

The following notation for equation (6.11) holds:

$$
\begin{aligned}
m_{1_{4}}^{(l)} & =\prod_{j=1}^{l-1} \lambda_{j} \sum_{j=1}^{l} \sum_{\substack{k=1 \\
k \neq j}}^{l} F_{j k} \\
& =\prod_{j=1}^{l-1} \lambda_{j} \sum_{j=1}^{l} \sum_{k>j}^{l}\left(F_{j k}+F_{k j}\right) .
\end{aligned}
$$

Factor $F_{j k}$ is given as:

$$
F_{j k}=\exp \left(-\lambda_{j} t\right)\left(\prod_{\substack{k=1 \\ k \neq j}}^{l} \frac{1}{\lambda_{k}-\lambda_{j}}\right)\left(\prod_{\substack{l=1 \\ l \neq k \\ l \neq j}}^{l} \frac{\lambda_{j l}}{\lambda_{j l}-\lambda_{j k}}\right)\left(b-\frac{a}{\lambda_{j k}}\right) \frac{1}{\lambda_{j k}} \exp \left(-\lambda_{j k}\left(1-v_{j} t\right)\right),
$$

where factor $F_{k j}$ emerges by interchanging the indices $j$ and $k$.

Subsequently we simplify equation (6.12). The pairwise factors $F_{j k}$ and $F_{k j}$ can be eliminated by

$$
F_{j k}+F_{k j}=0
$$


with $j=1, \ldots, i$ and $k>j$. Then there holds for term $m_{1_{4}}^{(l)}$ :

$$
m_{14}^{(l)}=0
$$

Hence, we can write the analytical solution of the remaining mass as given in (6.4).

For the outflowing mass $m_{2}^{l}$ we compute the summation of the masses $m_{\text {ges }}^{l}$.

Theorem 6.2. For the summation of the mass, we have the analytical solution given as

$$
\begin{aligned}
m_{\text {ges }}^{(l)} & =m_{\text {impuls }} c_{G D G L}^{(l)} \\
& =m_{1}^{(l)}+m_{2}^{(l)} .
\end{aligned}
$$

The total mass is given as

$$
m_{\mathrm{ges}}^{(l)}=\Lambda^{(l)}\left(\sum_{i=1}^{l} \Lambda_{i}^{(l)} \exp \left(-\lambda_{i} t\right)\left(a \frac{1}{2}+b\right)\right)
$$

Proof. The outline of the proof is adduced as in Theorem 6.1 and details are given in [18].

The outflowing mass is calculated as

$$
m_{2}^{(l)}=m_{\text {ges }}^{(l)}-m_{1}^{(l)} .
$$

For the discretization we get the following functions:

$$
\begin{aligned}
& m_{i j, \text {,rest }}^{(l), n}\left(\tau^{n}\right)=m_{1}^{(l)}\left(a, b, \tau^{n}, v_{1, i}, \ldots, v_{l, i}, R^{(1)}, \ldots, R^{(l)}, \lambda^{(1)}, \ldots, \lambda^{(l)}\right), \\
& m_{i j, \text { out }}^{(l), n}\left(\tau^{n}\right)=m_{2}^{(l)}\left(a, b, \tau^{n}, v_{1, i}, \ldots, v_{l, i}, R^{(1)}, \ldots, R^{(l)}, \lambda^{(1)}, \ldots, \lambda^{(l)}\right),
\end{aligned}
$$

where $a=R_{i}^{(l)} V_{i}\left(c_{i j}^{(l), n}\left(\tau^{n}\right)-c_{i j^{\prime}}^{(l), n}\right), b=R_{i}^{(l)} V_{i} c_{i j^{\prime}}^{(l), n}$ and $j \in \operatorname{out}(i)$.

For equations (6.17) and (6.18) we have used the one-dimensional outflow through the outflown boundary, given as

$$
\nu_{i}=v_{i j}, j=\operatorname{out}(i),
$$

where the CFL condition, that has to be fulfilled, is given as

$$
\tau_{l, i}=\frac{V_{i} R_{i}^{(l)}}{\nu_{i}}
$$

Therefore the velocity for each cell $i$ on the unit interval is given as

$$
v_{l, i}=\frac{1}{\tau_{l, i}}
$$

Thus the resulting time-step, used in (6.17) and (6.18), is given as $\tau^{n} \leq \min _{\substack{l=1, \ldots, M \\ i=1, \ldots, I}}\left\{\tau_{l, i}\right\}$.

For an illustration of the piecewise linear impulse applied for the mass solution in (6.4) refer to Figure 3. 


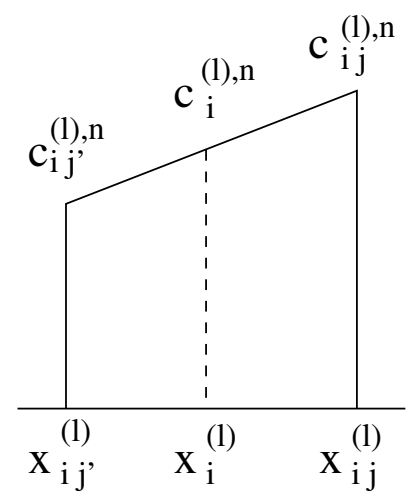

FIGURE 3. Piecewise linear impulse given with the concentrations $c_{i j}^{(l), n}\left(\tau^{n}\right)$ and $c_{i j^{\prime}}^{(l), n}$.

\section{Diffusion-Dispersion DisCRETIZATION SCHEME}

We discretize the diffusion-dispersion equation with implicit time discretization and finite volume methods with constant test functions. We deal with the diffusion-dispersion equation given as

$$
\partial_{t} R u-\nabla \cdot(D \nabla u)=0
$$

where $u=u(x, t)$ with $x \in \Omega$ and $t \geq 0$. The diffusions-dispersions tensor is $D=D(x, \boldsymbol{v})$ given by the Scheidegger approach ( $c f$. [32]). The velocity is given by $\boldsymbol{v}$ and is piecewise constant in the cells. The retardation factor is $R>0$.

We have Neumann boundary values, given as $\mathbf{n} \cdot D \nabla u(x, t)=0$, where $x \in \Gamma=\partial \Omega, c f$. [15]. The initial conditions are given as $u(x, 0)=u_{0}(x)$.

We integrate equation (7.1) over space and time and get

$$
\int_{\Omega_{j}} \int_{t^{n}}^{t^{n+1}} \partial_{t} R(u) \mathrm{d} t \mathrm{~d} x=\int_{\Omega_{j}} \int_{t^{n}}^{t^{n+1}} \nabla \cdot(D \nabla u) \mathrm{d} t \mathrm{~d} x .
$$

We apply the backward Euler method and exactly integrate the left hand side of equation (7.2). The right-hand side is lumped for the diffusion-dispersion term, $c f$. [18], thus there holds:

$$
\int_{\Omega_{j}}\left(R\left(u^{n+1}\right)-R\left(u^{n}\right)\right) \mathrm{d} x=\tau^{n} \int_{\Omega_{j}} \nabla \cdot\left(D \nabla u^{n+1}\right) \mathrm{d} x .
$$

Equation (7.3) is discretized over the space with Green's formula. We obtain the following equation

$$
\int_{\Omega_{j}}\left(R\left(u^{n+1}\right)-R\left(u^{n}\right)\right) \mathrm{d} x=\tau^{n} \int_{\Gamma_{j}} D \mathbf{n} \cdot \nabla u^{n+1} \mathrm{~d} \gamma
$$

where $\Gamma_{j}$ is the boundary of the finite volume cell $\Omega_{j}$. We use the approximation in space (cf. [18]).

The integration of equation (7.4) is done for finite boundaries. Using the middle-point rule yields:

$$
V_{j} R\left(u_{j}^{n+1}\right)-V_{j} R\left(u_{j}^{n}\right)=\tau^{n} \sum_{e \in \Lambda_{j}} \sum_{k \in \Lambda_{j}^{e}}\left|\Gamma_{j k}^{e}\right| \mathbf{n}_{j k}^{e} \cdot D_{j k}^{e} \nabla u_{j k}^{e, n+1},
$$


where $\left|\Gamma_{j k}^{e}\right|$ is the length of the boundary element $\Gamma_{j k}^{e}$. The gradients are calculated with the piecewise finite element function $\phi_{l}$ and we get

$$
\nabla u_{j k}^{e, n+1}=\sum_{l \in \Lambda^{e}} u_{l}^{n+1} \nabla \phi_{l}\left(\mathbf{x}_{j k}^{e}\right) .
$$

Because of the reconstruction of the gradient with the piecewise linear finite element functions, we obtain a second-order discretization method ( $c f .[16])$. This leads to the following discretization form:

$$
V_{j} R\left(c_{j}^{n+1}\right)-V_{j} R\left(c_{j}^{n}\right)=\tau^{n} \sum_{e \in \Lambda_{j}} \sum_{l \in \Lambda^{e} \backslash\{j\}}\left(\sum_{k \in \Lambda_{j}^{e}}\left|\Gamma_{j k}^{e}\right| \mathbf{n}_{j k}^{e} \cdot D_{j k}^{e} \nabla \phi_{l}\left(\mathbf{x}_{j k}^{e}\right)\right)\left(u_{j}^{n+1}-u_{l}^{n+1}\right),
$$

where $j=1, \ldots, m$.

In the next section we describe the operator-splitting methods, that are used to decouple the full equations.

\section{Operator-Splitting Methods}

Operator-splitting methods are used to decouple complicated partial differential equations into simpler equations and are often used in the geophysical and environmental physics. They are developed and applied in $[36,39,40]$.

The idea in this article is to solve simpler equations with higher-order discretization methods. For this purpose we use the operator-splitting method and decouple the equation with respect to the different time scales into simpler equations. There are many possible ways to combine ways to achieve an effective higher-order discretization method. One possibility is to discretize the convection equation with a characteristic method, the diffusion-dispersion equations with finite volume methods, and the reaction equations with exact methods. Another way would be to solve the convection-reaction equations with one mixed discretization method, and the diffusion-dispersion equation with finite volume methods.

In the following we consider the system of ordinary differential equations given as

$$
\partial_{t} u(t)=A u(t)+B u(t),
$$

where the initial conditions are $u^{n}=u\left(t^{n}\right)$. The operators $A$ and $B$ are assembled by the spatial discretizations, e.g. the convection part with characteristic methods and the diffusion part with finite volume methods.

The Lie-Trotter splitting is an operator-splitting method, which is of first-order for the non-commutative case. It solves two equation parts sequentially with respect to the initial conditions (see $[29,35]$ ). We get two simpler equations

$$
\begin{aligned}
& \frac{\partial u^{*}(t)}{\partial t}=A u^{*}(t), \quad \text { with } u^{*}\left(t^{n}\right)=u^{n}, \\
& \frac{\partial u^{* *}(t)}{\partial t}=B u^{* *}(t), \quad \text { with } u^{* *}\left(t^{n}\right)=u^{*}\left(t^{n+1}\right),
\end{aligned}
$$

where the time-step is $\tau^{n}=t^{n+1}-t^{n}$. The solutions of the equations are $u^{n+1}=u^{* *}\left(t^{n+1}\right)$.

The local splitting error of the sequential splitting method is given as $(c f .[18])$ :

$$
\rho_{n}=\frac{1}{2} \tau^{n}[A, B] u\left(t^{n}\right)+O\left(\left(\tau^{n}\right)^{2}\right),
$$

where $[A, B]:=A B-B A$ is the commutator of $A$ and $B$. We get an error $O\left(\tau^{n}\right)$, if the operators $A$ and $B$ do not commute, otherwise the method is exact.

We improve our method by the so-called Strang splitting method, which is of second-order (cf. [36]). 
The method is presented as

$$
\begin{aligned}
& \frac{\partial u^{*}(t)}{\partial t}=A u^{*}(t), \text { with } t^{n} \leq t \leq t^{n+1 / 2}, u^{*}\left(t^{n}\right)=u^{n}, \\
& \frac{\partial u^{* *}(t)}{\partial t}=B u^{* *}(t), \text { with } t^{n} \leq t \leq t^{n+1}, u^{* *}\left(t^{n}\right)=u^{*}\left(t^{n+1 / 2}\right), \\
& \frac{\partial u^{* * *}(t)}{\partial t}=A u^{* * *}(t), \text { with } t^{n+1 / 2} \leq t \leq t^{n+1}, u^{* * *}\left(t^{n+1 / 2}\right)=u^{* *}\left(t^{n+1}\right),
\end{aligned}
$$

where the results of the method are $u^{n+1}=u^{* * *}\left(t^{n+1}\right)$.

The splitting error of this method is given as (cf. [24]),

$$
\rho_{n}=\frac{1}{24}\left(\tau^{n}\right)^{2}([B,[B, A]]-2[A,[A, B]]) u\left(t^{n}\right)+O\left(\left(\tau^{n}\right)^{4}\right)
$$

where we get the second-order for non-commuting operators.

For our methods it is sufficient to have second-order decomposition methods. Further higher-order splitting methods are described in the literature $[11,21,25]$.

In the next section we present the results of our numerical experiments.

\section{NumericAl EXPERIMENTS}

\subsection{Benchmark model: one-dimensional example}

In the first example, we illustrate the method used in Section 6.1 on a simple 1D convection-reaction equation with four components, where the porosity is $\phi$ and the retardation factors have the values $R^{(1)}=1, R^{(2)}=2$, $R^{(3)}=4$ and $R^{(4)}=8$. The numerical solution at the last computation time $t=6$ is presented in Figure 4 and it cannot be distinguished from the exact solution. Here, we can apply the characteristics method for the time-discretisation, which is exact, and the second-order finite volume methods for the spatial-discretization. The computations are realized with $\tau^{n} \tau_{\mathrm{CFL}}^{n} / 2$, i.e. with the Courant number 0.5 , the domain $\Omega$ is given as the interval $(0,8) \subset \mathbb{R}^{+}$.

In Table 1, we compare the absolute error $E_{1}$ for the standard operator-splitting approach (the second column) and the equivalently defined error $E_{2}$ for the new algorithm (the fourth column). The results are presented for several uniformly refined grids and the numerical convergence rates are presented for the operator-splitting algorithm (the third column) and the new algorithm (the fifth column). As expected, for the operator-splitting method, the convergence rate is approximately one, i.e. the absolute error is halved for one grid refinement, and the convergence rate for the new algorithm is approximately two, i.e. the absolute error is four times smaller after one grid refinement.

In the next subsection we present complex scenarios in a waste disposal done in a salt dome.

\subsection{Benchmark model: two-dimensional example}

In the second example, we illustrate a benchmark problem, that is also discussed as a Couplex benchmark problem in the literature, see $[2,5]$.

Here we apply a version with analytical solutions which simulate a simple waste-disposal.

We simulate a realistic potential damage event in a repository with radioactive contaminates. Again the convection-reaction equation is considered as a dominant model problem for long-distance flow, the so-called fare-field model, see [2].

The realistic parameters were used for a long-term analysis of the repository. Exchange and retardation processes were simulated.

The numerical results were compared with the analytical solutions and therefore the method was also examined for realistic parameters. 


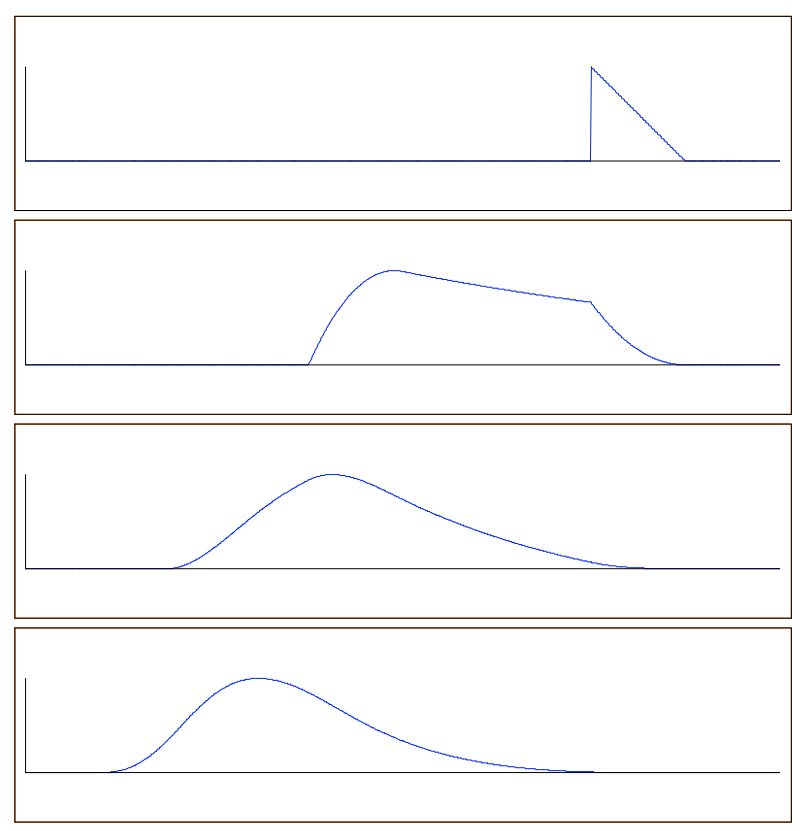

FiguRE 4. Four components of the numerical solution for $t=6$ with the retardation factors $R^{(1)}=1$ (top), $R^{(2)}=2, R^{(3)}=4$ and $R^{(4)}=8$ (bottom). The velocity is $v \equiv 1$ and the domain is $(0,8) \subset R$.

TABLE 1. The absolute error $E_{1}$ (operator-splitting method) and $E_{2}$ (the new algorithm) for the fourth component of the numerical solution. The third and fifth columns contain the corresponding convergence rates.

\begin{tabular}{|c||c|c||c|c|}
\hline$h$ & $E_{1} \cdot 10^{-4}$ & $\alpha_{1}$ & $E_{2} \cdot 10^{-4}$ & $\alpha_{2}$ \\
\hline \hline $1 / 16$ & 413.2 & & 23.76 & \\
$1 / 32$ & 201.3 & 1.04 & 5.573 & 2.09 \\
$1 / 64$ & 99.25 & 1.02 & 1.374 & 2.02 \\
$1 / 128$ & 49.25 & 1.01 & 0.344 & 1.99 \\
\hline
\end{tabular}

The calculations are a preliminary stage in complex model geometries, on which the methods are applied. Hence, the descent on multi-dimensional model geometries was prepared.

The decay chain in the following example is taken from the neptunium sequence. We used this decay chain, since the retardation and decay factors are vary considerably over multiple decimal powers.

The decay chain is denoted by:

$$
\mathrm{Am}-241 \rightarrow \mathrm{Np}-237 \rightarrow \mathrm{U}-233 \rightarrow \mathrm{Th}-229
$$

The retardation factors are:

$$
R_{\mathrm{Am}}=200.2, R_{\mathrm{Np}}=2.2, R_{\mathrm{U}}=1.6, R_{\mathrm{Th}}=2000.2 \text {. }
$$

The porosity is:

$$
\phi=0.5 \text {. }
$$


TABLE 2. Absolute $L_{1}$-error for the standard method with one-dimensional model problem and realistic parameters.

\begin{tabular}{|c||c|c|c|c|}
\hline$l$ & $E_{L_{1}}^{1}$ & $E_{L_{1}}^{2}$ & $E_{L_{1}}^{3}$ & $E_{L_{1}}^{4}$ \\
\hline \hline 4 & $4.13 \times 10^{-1}$ & $1.96 \times 10^{6}$ & $2.71 \times 10^{3}$ & $3.14 \times 10^{-4}$ \\
5 & $1.59 \times 10^{-1}$ & $8.62 \times 10^{5}$ & $1.31 \times 10^{3}$ & $9.55 \times 10^{-5}$ \\
6 & $5.66 \times 10^{-2}$ & $4.28 \times 10^{5}$ & $6.52 \times 10^{2}$ & $4.20 \times 10^{-5}$ \\
7 & $2.10 \times 10^{-2}$ & $2.15 \times 10^{5}$ & $3.26 \times 10^{2}$ & $1.66 \times 10^{-5}$ \\
\hline
\end{tabular}

TABLE 3 . Orders of convergence for $L_{1}$-errors for the standard method with one-dimensional model problem and realistic parameters.

\begin{tabular}{|c||c|c|c|c|}
\hline$l$ & $\rho_{L_{1}}^{1}$ & $\rho_{L_{1}}^{2}$ & $\rho_{L_{1}}^{3}$ & $\rho_{L_{1}}^{4}$ \\
\hline \hline 4 & & & & \\
5 & 1.58 & 1.18 & 1.04 & 1.7 \\
6 & 1.44 & 1.01 & 1.006 & 1.18 \\
7 & 1.43 & 0.99 & 1.0 & 1.2 \\
\hline
\end{tabular}

The half-life periods are:

$$
\begin{aligned}
& T_{1 / 2, \mathrm{Am}}=1.3639 \times 10^{10}[\mathrm{~s}], T_{1 / 2, \mathrm{~Np}}=6.7659 \times 10^{13}[\mathrm{~s}], \\
& T_{1 / 2, \mathrm{U}}=15.0239 \times 10^{12}[\mathrm{~s}], T_{1 / 2, \mathrm{Th}}=2.4867 \times 10^{11}[\mathrm{~s}] .
\end{aligned}
$$

For the simulation we consider a time period of 6340 years. The time-step width is calculated with a time-step control and the limitation by the Courant number 0.5 , see equation (4.12).

The model area is 8000 times $1000 \mathrm{~m}$.

The initial conditions are given with a triangular impulse with length $500 \mathrm{~m}$. The impulse trends over the total width of the area. Therefore the test case degenerates to a one-dimensional example. The initial concentrations are given with 1.0.

The boundary conditions are trivial inflow and outflow boundary conditions, where $\mathbf{n} \cdot \mathbf{v} c=0$ holds.

No inflow sources occur, i.e. $\tilde{Q}_{i}=0.0$ for $i=1, \ldots, 4$.

The velocity is one-dimensional along the $x$-direction and constant in the area with $\mathbf{v}=\left(2 \times 10^{-8}, 0\right)^{T}\left[\mathrm{~m} \cdot s^{-1}\right]$.

For the evaluation of the numerical calculations with error norms and orders of convergence, many calculations were realized with varying grid levels.

The calculations of the standard and modified method are compared.

\section{Standard method}

In the subsequent evaluations we calculate the numerical solutions with the established standard method. The errors are calculated with the $L_{1}$-norm. The absolute error between numerical and analytical solution is quoted.

The large-scale differences of the parameters, which reach over some decimal powers, spread to the absolute errors of the components. The results of the absolute errors are listed in Table 2 .

The experimental order of convergence can be calculated with the values of absolute errors from Table 2 . The orders of convergence are given in Table 3.

Based on the smooth initial impulse for the first component, the order of convergence is of higher-order. The further components have the order of convergence 1 based on the usage of the standard method. The standard method yields worse results for higher components.

An improvement in the results can be obtained with the modified method and is considered subsequently. 
TABLE 4. Absolute $L_{1}$-error for the modified method with one-dimensional model problem and realistic parameters.

\begin{tabular}{|c||c|c|c|c|}
\hline$l$ & $E_{L_{1}}^{1}$ & $E_{L_{1}}^{2}$ & $E_{L_{1}}^{3}$ & $E_{L_{1}}^{4}$ \\
\hline \hline 4 & $4.13 \times 10^{-1}$ & $1.19 \times 10^{6}$ & $3.188 \times 10^{2}$ & $4.355 \times 10^{-4}$ \\
5 & $1.54 \times 10^{-1}$ & $2.46 \times 10^{5}$ & $5.33 \times 10^{1}$ & $5.66 \times 10^{-5}$ \\
6 & $5.66 \times 10^{-2}$ & $8.92 \times 10^{4}$ & $1.13 \times 10^{1}$ & $1.02 \times 10^{-5}$ \\
7 & $2.10 \times 10^{-2}$ & $2.35 \times 10^{4}$ & $2.89 \times 10^{0}$ & $2.89 \times 10^{-6}$ \\
\hline
\end{tabular}

TABLE 5. Orders of convergence of $L_{1}$-error for the modified method with one-dimensional model problem and realistic parameters.

\begin{tabular}{|c||c|c|c|c|}
\hline$l$ & $\rho_{L_{1}}^{1}$ & $\rho_{L_{1}}^{2}$ & $\rho_{L_{1}}^{3}$ & $\rho_{L_{1}}^{4}$ \\
\hline \hline 4 & & & & \\
5 & 1.58 & 2.27 & 2.58 & 2.94 \\
6 & 1.44 & 1.58 & 2.23 & 2.47 \\
7 & 1.43 & 1.93 & 1.967 & 1.72 \\
\hline
\end{tabular}

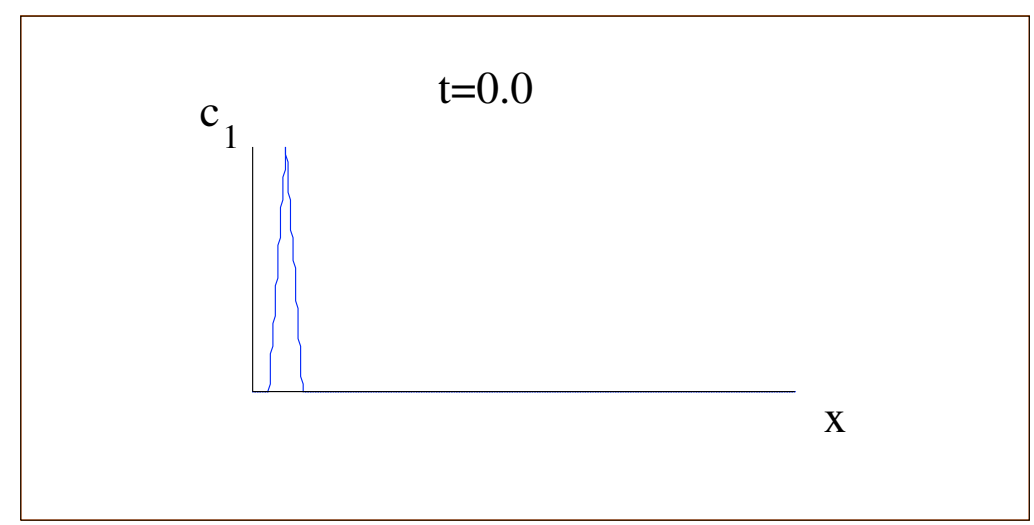

FiguRE 5. Initialization of first component at time $t=0[a]$.

\section{Modified method}

The numerical calculations with the modified method are compared with the analytical solutions and evaluated using the $L_{1}$-error. Table 4 shows the errors of the calculations with the modified method.

The experimental orders of convergence are listed in Table 5 for the modified method.

The modified method confirms the results for higher-orders of convergence based on the improved method. The method can confirm the theoretical results for the higher-order of the discretization for particularly scaledependent model problems, as they occur in realistic models.

The results are visualized graphically. The initial conditions are shown in Figure 5 for the first component.

The further illustrations show the concentrations at end time $6340[a]$.

The progress of the impulses is visualized in Figure 6. The first component has the strongest retardation and is least transported. The second and third components have the smallest retardation and are transported furthermost. Based on the coupling to the first component an expansion of the impulses up to the first component emerges. The fourth component again is very strongly retarded and is sparsely transported. Based on the coupling and because of the decay with the predecessor, the impulse is expanded over the whole length up to the preceding concentration. 

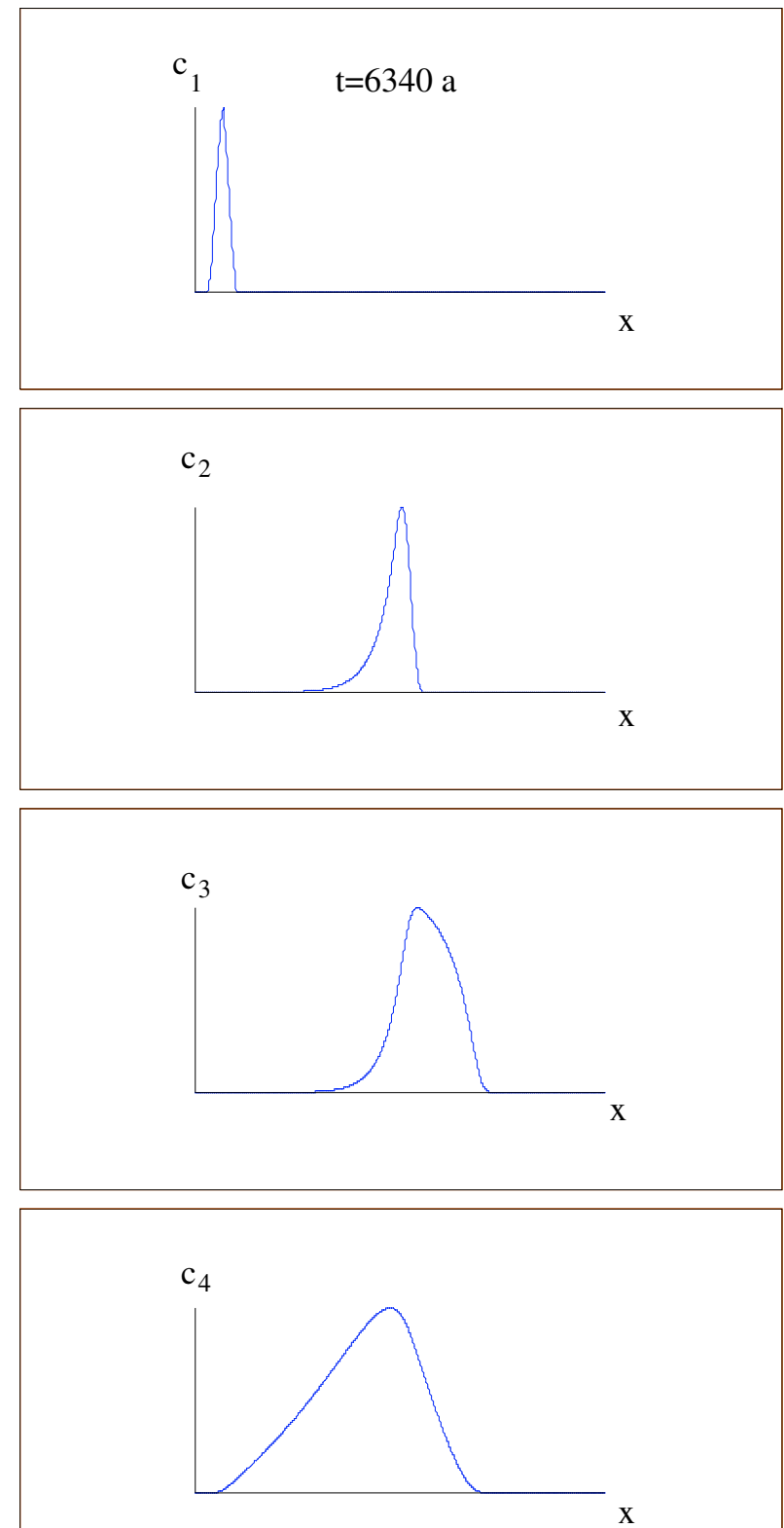

FiguRE 6. Expansion of contaminant after 6340 years.

A further visualization is given in Figure 7. It shows the expansion of the contaminant in a color plot. The red area expresses the maximal concentration of components, the blue area illustrates the minimal concentration of components.

\subsection{Real-life problem: two-dimensional model of a waste disposal}

We calculate some scenarios of waste cases, which helps us to get new conclusions about the waste disposals in salt domes. 

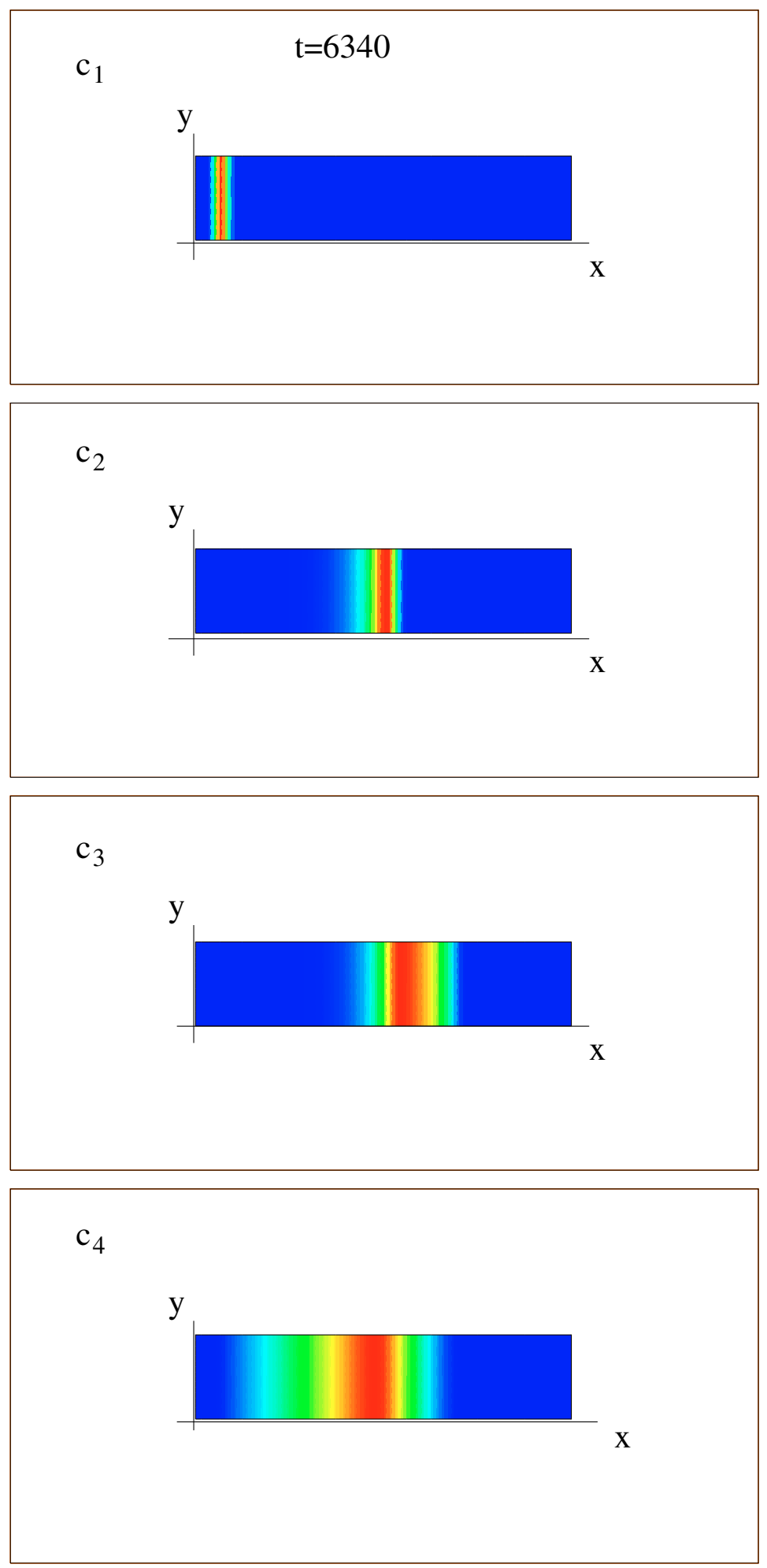

FiguRE 7. Expansion of contaminant after 6340 years with color plot. 


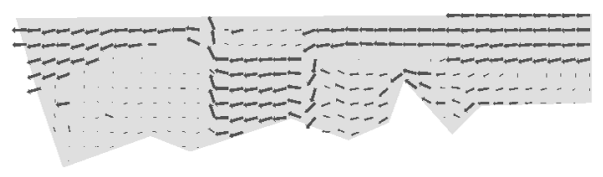

Figure 8. Flow field for a two-dimensional calculation.

We have a model based on an overlying rock over a salt dome. We suppose a waste case such that a permanent source of radioactive contaminant groundwater flows from the bottom of the overlying rock, where the waste disposal is situated. We suppose that the contaminants are flown with the groundwater, which is flown through the overlying rock. From our model we calculate the transport and the reaction of this contaminant coupled with decay chains. The simulation time is 10000 [a] and we calculate the concentration that has flown up to the top of the overlying rock. With these dates we can conclude whether the waste disposal is safe enough. The two-dimensional test case is presented with the dates of our project partner GRS in Braunschweig (Germany), cf. $[12,13]$.

We have a model domain with dimensions of $6000[\mathrm{~m}] \times 150[\mathrm{~m}]$ with four different layers with different permeabilities, see [12]. The domain is spooled with groundwater from the right boundary to the left boundary. The groundwater is flowing faster through the permeable layer than through the impermeable layers. Therefore the groundwater flows from the right boundary to the half middle of the domain. It is flowing through the permeable layer down to the bottom of the domain and is spooled up in the left domain to the top. The groundwater flows in the left top part to the outflow at the left boundary. The flow field with the velocity is calculated with the program package $\mathbf{d}^{\mathbf{3}} \mathbf{f}$ and presented in Figure 8. In the middle of the bottom of the domain, the contaminants are flown in as a permanent source. With the stationary velocity field, the contaminants are computed with the software package $R^{3} T$. The flow field transports the radioactive contaminants up to the top of the domain. The decay chain is presented with 26 components as follows

$$
\begin{aligned}
& \mathrm{Pu}-244 \rightarrow \mathrm{Pu}-240 \rightarrow \mathrm{U}-236 \rightarrow \mathrm{Th}-232 \rightarrow \mathrm{Ra}-228 \\
& \mathrm{Cm}-244 \rightarrow \mathrm{Pu}-240 \\
& \mathrm{U}-232 \\
& \mathrm{Pu}-241 \rightarrow \mathrm{Am}-241 \rightarrow \mathrm{Np}-237 \rightarrow \mathrm{U}-233 \rightarrow \mathrm{Th}-229 \\
& \mathrm{Cm}-246 \rightarrow \mathrm{Pu}-242 \rightarrow \mathrm{U}-238 \rightarrow \mathrm{U}-234 \rightarrow \mathrm{Th}-230 \rightarrow \\
& \mathrm{Ra}-226 \rightarrow \mathrm{Pb}-210 \\
& \mathrm{Am}-242 \rightarrow \mathrm{Pu}-238 \rightarrow \mathrm{U}-234 \\
& \mathrm{Am}-243 \rightarrow \mathrm{Pu}-239 \rightarrow \mathrm{U}-235 \rightarrow \mathrm{Pa}-231 \rightarrow \mathrm{Ac}-227 .
\end{aligned}
$$

We present the important concentration in this decay chain. In Figure 9 the contaminant uranium isotope $\mathrm{U}-236$ is presented after 100 [a]. This isotope is less retarded and has a very long half-life period. Therefore the contaminant is flown furthermost and decays less. This effect is presented in Figure 9. The diffusion process has spread out the contaminant in the whole left part of the domain. The impermeable layer is also contaminated. After the time period of 10000 [a], the contaminant is flown up to the top of the domain.

The calculations are done on uniform grids. The convergence of these grids is confirmed with adaptive grid calculations. The calculations confirm the results of finer and smaller time-steps (cf. Tab. 6). The early calculations are done by explicit methods until the character of the equation is more diffusive. Then we move to the implicit methods and can use larger time-steps. With this procedure we can fulfill the forced maximum calculation time of one day.

Our paper concludes with the next section. 

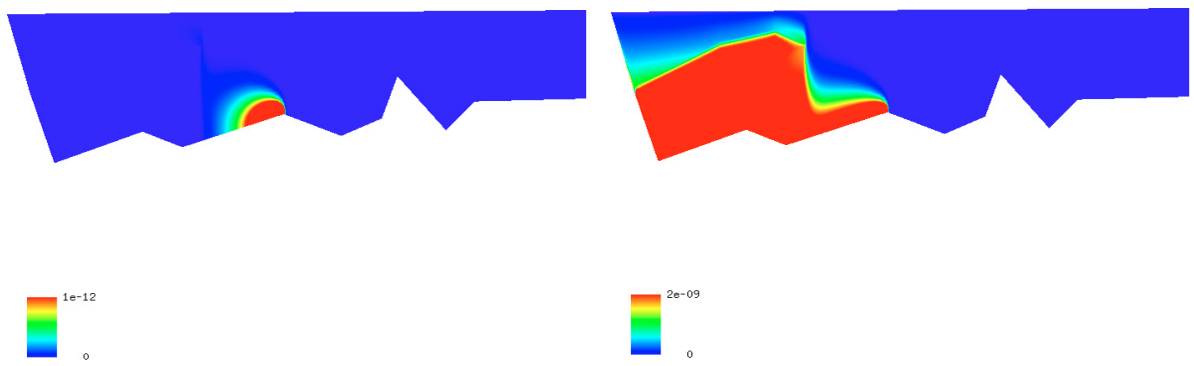

Figure 9. Concentration of $\mathrm{U}-236$ at the time point $t=100[\mathrm{a}]$ and $t=10000[\mathrm{a}]$.

TABLE 6. Computing the two-dimensional case.

\begin{tabular}{|c||c|c|c|c|c|}
\hline Processors & Refinement & $\begin{array}{c}\text { Number of } \\
\text { elements }\end{array}$ & $\begin{array}{c}\text { Number of } \\
\text { time-steps }\end{array}$ & $\begin{array}{c}\text { Time for } \\
\text { one time-step }\end{array}$ & $\begin{array}{c}\text { Total } \\
\text { time }\end{array}$ \\
\hline \hline 30 & uniform & 75000 & 3800 & $5 \mathrm{~s}$ & $5.5 \mathrm{~h}$ \\
\hline \hline 64 & adaptive & 350000 & 3800 & $14 \mathrm{~s}$ & $14.5 \mathrm{~h}$ \\
\hline
\end{tabular}

\section{Conclusion AND Discussion}

We present discretization methods to solve a complex system of advection-diffusion-reaction equations. Using the finite volume methods we present improved discretization methods for convection, diffusion-dispersion and convection-reaction equations. With a new embedding method for the analytical solutions we improve the discretization methods for the convection-reaction equations and we can skip the error in the temporal discretization. Second-order operator-splitting methods allow us to decouple the full equation system into simpler equations and to discretize each of them with higher-order finite volume methods. We verify the theoretical results with benchmark applications. Realistic test examples and complex waste scenarios are presented. We can confirm, that a complex model could be simulated with the help of different splitting and discretization methods.

In the future we will focus on the development of improved discretization methods and the idea of embedding local analytical solutions with decoupling into simpler physical processes.

\section{REFERENCES}

[1] T. Barth and M. Ohlberger, Finite volume methods: foundation and analysis, in Encyclopedia of Computational Mechanics, E. Stein, R. de Borst and T.J.R. Hughes Eds., John Wiley \& Sons, Ltd (2004).

[2] P. Bastian and S. Lang, Couplex benchmark computations with UG. Computat. Geosci. 8 (2004) 125-147.

[3] J. Bear, Dynamics of fluids in porous media. American Elsevier, New York, USA (1972).

[4] J. Bear and Y. Bachmat, Introduction to Modeling of Transport Phenomena in Porous Media. Kluwer Academic Publishers, Dordrecht, Boston, London (1991).

[5] A. Bourgeat, M. Kern, S. Schumacher and J. Talandier, The COUPLEX test cases: Nuclear waste disposal simulation: Simulation of transport around a nuclear waste disposal site. Computat. Geosci. 8 (2004) 83-98.

[6] M.A. Celia, T.F. Russell, I. Herrera and R.E. Ewing, An Eulerian-Lagrangian localized adjoint method for the advectiondiffusion equation. Adv. Wat. Res. 13 (1990) 187-206.

[7] G.R. Eykolt, Analytical solution for networks of irreversible first-order reactions. Wat. Res. 33 (1999) 814-826.

[8] R. Eymard, T. Gallouët and R. Herbin, Finite volume methods, in Handbook of Numerical Analysis 7, Amsterdam, North Holland (2000) 713-1020.

[9] R. Eymard, T. Gallouët and R. Herbin, Finite volume approximation of elliptic problems and convergence of an approximate gradient, in Handbook of Numerical Analysis 37, Appl. Numer. Math. (2001) 31-53. 
[10] R. Eymard, T. Gallouët and R. Herbin, Error estimates for approximate solutions of a nonlinear convection-diffusion problem. Adv. Differ. Equ. 7 (2002) 419-440.

[11] I. Farago and J. Geiser, Iterative operator-splitting methods for linear problems. International J. Computat. Sci. Eng. 3 (2007) $255-263$.

[12] E. Fein, Test-example for a waste-disposal and parameters for a decay-chain. Private communications, Braunschweig, Germany (2000).

[13] E. Fein, Physical Model and Mathematical Description. Private communications, Braunschweig, Germany (2001).

[14] E. Fein, T. Kühle and U. Noseck, Development of a software-package for three dimensional models to simulate contaminated transport problems. Technical Concepts, Braunschweig, Germany (2001).

[15] P. Frolkovič, Flux-based method of characteristics for contaminant transport in flowing groundwater. Comput. Vis. Sci. 5 (2002) 73-83.

[16] P. Frolkovič and H. De Schepper, Numerical modeling of convection dominated transport coupled with density driven flow in porous media. Adv. Wat. Res. 24 (2001) 63-72.

[17] P. Frolkovič and J. Geiser, Numerical Simulation of Radionuclides Transport in Double Porosity Media with Sorption, in Proceedings of Algorithmy 2000, Conference of Scientific Computing (2000) 28-36.

[18] J. Geiser, Gekoppelte Diskretisierungsverfahren für Systeme von Konvektions-Dispersions-Diffusions-Reaktionsgleichungen. Doktor-Arbeit, Universität Heidelberg, Germany (2004).

[19] M.T. Genuchten, Convective-dispersive transport of solutes involved in sequential first-order decay reactions. Comput. Geosci. 11 (1985) 129-147.

[20] S.K. Godunov, A finite difference method for the numerical computation of discontinuous solutions of the equations of fluid dynamics. Mat. Sb. 47 (1959) 271-290.

[21] E. Hairer, C. Lubich and G. Wanner, Geometric Numerical Integration: Structure-Preserving Algorithms for Ordinary Differential Equations, Springer Series in Computational Mathematics 31. Springer Verlag Berlin, Heidelberg, New York (2002).

[22] A. Harten, B. Enguist, S. Osher and S. Charkravarthy, Uniformly high order esssentially non-oscillatory schemes I. SIAM J. Numer. Anal. 24 (1987) 279-309.

[23] A. Harten, B. Enguist, S. Osher and S. Charkravarthy, Uniformly high order esssentially non-oscillatory schemes III. J. Computat. Phys. 71 (1987) 231-303.

[24] W.H. Hundsdorfer, Numerical Solution of Advection-Diffusion-Reaction Equations. Technical Report NM-N9603, CWI (1996).

[25] W. Hundsdorfer and J.G. Verwer, Numerical Solution of Time-dependent Advection-Diffusion-Reaction Equations, Springer Series in Computational Mathematics 33. Springer Verlag (2003).

[26] X.-D. Liu, S. Osher and T. Chan, Weighted essentially non-oscillatory schemes. J. Comput. Phys. 115 (1994) $200-212$.

[27] R.J. LeVeque, Numerical Methods for Conservation Laws, Lectures in Mathematics. Birkhäuser Verlag Basel, Boston, Berlin (1992).

[28] R.J. LeVeque, Finite Volume Methods for Hyperbolic Problems, Cambridge Texts in Applied Mathematics. Cambridge University Press (2002).

[29] R.I. McLachlan, R.G.W. Quispel, Splitting methods. Acta Numer. 11 (2002) 341-434.

[30] K.W. Morton, On the analysis of finite volume methods for evolutionary problems. SIAM J. Numer. Anal. 35 (1998) $2195-2222$.

[31] P.J. Roache, A flux-based modified method of characteristics. Int. J. Numer. Methods Fluids 12 (1992) 1259-1275.

[32] A.E. Scheidegger, General theory of dispersion in porous media. J. Geophysical Research 66 (1961) 32-73.

[33] C.-W. Shu, High order finite difference and finite volume WENO schemes and discontinuous Galerkin methods for CFD. Internat. J. Comput. Fluid Dynamics 17 (2003) 107-118.

[34] T. Sonar, On the design of an upwind scheme for compressible flow on general triangulation. Numer. Anal. 4 (1993) $135-148$.

[35] B. Sportisse, An analysis of operator-splitting techniques in the stiff case. J. Comput. Phys. 161 (2000) 140-168.

[36] G. Strang, On the construction and comparison of difference schemes. SIAM J. Numer. Anal. 5 (1968) 506-517.

[37] Y. Sun, J.N. Petersen and T.P. Clement, Analytical solutions for multiple species reactive transport in multiple dimensions. J. Contam. Hydrol. 35 (1999) 429-440.

[38] V. Thomee, Galerkin Finite Element Methods for Parabolic Problems, Lecture Notes in Mathematics 1054. Springer Verlag, Berlin, Heidelberg (1984).

[39] J.G. Verwer and B. Sportisse, A note on operator-splitting in a stiff linear case. MAS-R9830, ISSN (1998) 1386-3703.

[40] Z. Zlatev, Computer Treatment of Large Air Pollution Models. Kluwer Academic Publishers (1995). 